\title{
Symbols and quantities in parietal cortex: elements of a mathematical theory of number representation and manipulation
}

\author{
Stanislas Dehaene
}

In this chapter, I put together the first elements of a mathematical theory relating neurobiological observations to psychological laws in the domain of numerical cognition. The starting point is the postulate of a neuronal code whereby numerosity-the cardinal of a set of objects-is represented approximately by the firing of a population of numerosity detectors. Each of these neurons fires to a certain preferred numerosity, with a tuning curve which is a Gaussian function of the logarithm of numerosity. From this logGaussian code, decisions are taken using Bayesian mechanisms of log-likelihood computation and accumulation. The resulting equations for response times and errors in classical tasks of number comparison and same-different judgments are shown to tightly fit behavioral and neural data. Two more speculative issues are discussed. First, new chronometric evidence is presented supporting the hypothesis that the acquisition of number symbols changes the mental number line, both by increasing its precision and by changing its coding scheme from logarithmic to linear. Second, I examine how symbolic and nonsymbolic representations of numbers affect performance in arithmetic computations such as addition and subtraction.

\section{Introduction}

An ultimate goal of psychology is to provide lawful explanations of mental mechanisms in terms of a small set of rules, preferably framed in the language of mathematics, which capture the regularities present in human and animal behavior. Furthermore, those psychological laws should not remain stated solely at a descriptive level (although obtaining valid descriptive rules of behavior is usually an indispensable step on that road). Rather, they should be ultimately grounded in a neurobiological level of explanation, through a series of additional bridging laws linking the molecular, synaptic, cellular, and circuit levels with psychological representation and computations.

Are these ambitious goals out of reach? No. In the domain of perception and motor control, solid psychological and bridiging laws have been described, one of the most successful cases being signal detection theory. Furthermore, at a higher cognitive level, 
the work of Roger Shepard has suggested that internal cognitive processes of mental representation and transformation can be captured by elegant mathematical rules, including Bayesian principles of similarity and categorization (Shepard, 2001).

In the present chapter, I will suggest that a model inspired by those earlier successes can begin to capture the main regularities observed in a small domain of semantics: the representation of number. We now have a good mathematical theory of the format of representation by which numerical quantities are encoded mentally, as well as of the main laws by which these representations are used to generate behavior in simple identity judgments, comparisons and calculations. Furthermore, two spectacular advances in electrophysiology - the discovery of single neurons tuned to numerosity in the macaque monkey (Sawamura et al., 2002; Nieder et al., 2002; Nieder and Miller, 2003, 2004), and of neurons plausibly implementing random-walk accumulation models of decision (Gold and Shadlen, 2002)—have given us insight into the neuronal mechanisms from which those laws arise.

The mathematical principles which now constitute a sort of 'standard model' for numerical cognition have been described over the years by various people and in various publications (e.g. Shepard et al., 1975; Van Oeffelen and Vos, 1982; Link, 1990; Dehaene, 1992; Dehaene and Mehler, 1992; Gallistel and Gelman, 1992; Dehaene and Changeux, 1993; Cordes et al., 2001; Dehaene, 2002, 2003; Nieder and Miller, 2003; Piazza et al., 2004; Pica et al., 2004; Verguts et al., 2005; Barth et al., 2006; McCrink et al., 2006). One of the goals of this chapter is to present a single reference source for equations that are currently widely dispersed in the literature. Nevertheless, mathematically less sophisticated readers can skip all of these equations, because their main points are also explained in plain language in the text. The main purpose of this chapter, indeed, is to provide an accessible synthesis of this theory and to compare its predictions with reanalyses of a variety of data. I shall do so in a 'theory-first' manner, first presenting the mathematical principles and then some of the best evidence for or against them (although historically, of course, theory development occurred in reverse order).

As we shall see, our ability to capture a variety of findings with a small set of principles is impressive. Nevertheless, systematic integration of multiple data into a coherent framework also leads to the identification of two important unsolved problems: How does the coding of symbolic information differ from that of nonsymbolic information (e.g. representing the meaning of the word 'thirteen' versus the quantity represented by 13 dots)? And what are the mechanisms by which we compute operations of addition and subtraction?

\section{Numerosity representation}

We start by specifying how the cardinal of sets is represented mentally. The term numerosity is used to refer to the cardinal property of sets, and to distinguish it from culture-dependent numerals or number symbols such as the word 'three'. Many experiments indicate that humans and other animal species possess a refined mental representation of numerosity, even when the use of number symbols is not available (in animals, preverbal infants, 
or cultures with a reduced number lexicon) or is made impossible (e.g. by verbal interference in adults).

\section{Mathematical theory: log-Gaussian model}

The theory presented here (See Figure 24.1) postulates that each numerosity is represented internally by a noisy distribution of activation on an internal continuum or mental number line. Mathematically, the numerosity of a set of $n$ dots is represented internally by a Gaussian random variable $X$ (the internal representative of $n$ ) with mean $q(n)$ and with standard deviation $w(n)$. Those parameters altogether specify the nature and precision of the numerical code. In particular, the parameter $w(n)$ determines the internal variability or amount of noise in the coding scheme.

There are several possible choices for $q(n)$ and $w(n)$, but they are constrained by a strong empirical observation, the fact that Weber's law holds for numerosity stimuli. As further discussed below, Weber's law states that the minimal numerical change that can be discriminated increases in direct proportion to the magnitude of the numerosities involved. An alternative formulation is that numerosity discrimination depends only on the ratio of the numbers involved, not their absolute values.

The simplest theoretical postulate is that internal variability is the same for all of the represented numbers $(w(n)=w)$. In order for Weber's law to hold, the internal variable then has to vary as the logarithm of the represented numerosity $n(q(n)=\log (n))$. In this case, the probability distribution which specifies the likelihood that a number $n$ is represented, at a given moment, by a particular value $x$ of the internal random variable $X$ is given by:

$$
p(X \in[x, x+d x])=G\left(\frac{x-\log (n)}{w}\right) d x=\frac{e^{-\frac{(x-\log [n])^{2}}{2 w^{2}}}}{\sqrt{2 \pi} w} d x
$$

where $G$ is the normal curve. This equation simply means that a given input numerosity is represented, at different moments, by noisy values that tend to cluster around a location corresponding to $\log (n)$ on the number line. I will refer to this model as the $\log$ Gaussian model. Note that the model has a single free parameter, $w$, the internal Weber fraction that specifies the degree of precision of the internal quantity representation.

An alternative model supposes that the internal variable scales linearly with $n$, but with a variability that also scales linearly with $n(w(n)=w \times n, q(n)=n)$. This is called the scalar variability model. Both models make essentially identical predictions for discrimination and comparison behavior, differing only in subtle, second-order terms that relate to asymmetries in the response curves. ${ }^{1}$ Thus, for purposes of computing error rates and response times, one may use one or the other model, depending on which is mathematically more tractable. More controversial, however, is whether the models are equivalent at

1 When $w$ is close to zero, as is true in human adults $(w \sim 0.15-0.20)$, so that $\log (1+w) \sim \mathrm{w}$, even the quantitative values of $w$ obtained with the log-Gaussian model and with the scalar variability model are close to identical. 


\section{Stimulus of numerosity $n$}

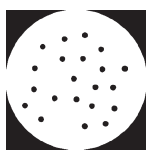

1. Coding by Log-Gaussian numerosity detectors

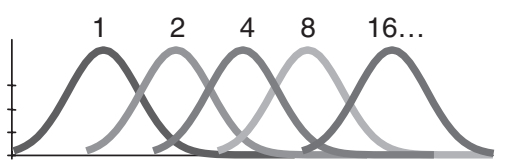

Internal logarithmic scale: $\log (n)$

2. Application of a criterion and formation of two pools of units

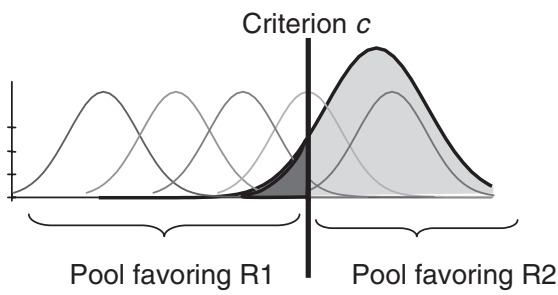

3. Computation of log-likelihood ratio by differencing

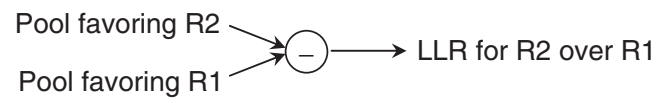

4. Accumulation of LLR, forming a random-walk process Mean Response Time

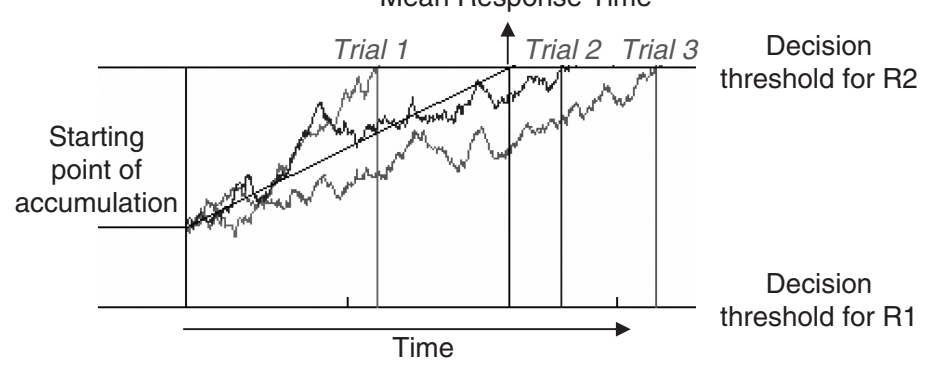

Figure 24.1 Overall outline of how the proposed mathematical theory accounts for elementary arithmetic decisions. Numerosity is coded by a fluctuating distribution of activation over log-Gaussian numerosity detectors. The decision is attained through to a random-walk process based on accumulation of estimates of the log-likelihood of the available responses. LLR, log-likelihood ratio.

all levels and, in particular, whether they can be distinguished at the neural level. Below, I will consider several subtle phenomena that seem to favor the logarithmic model.

\section{Neuronal modeling}

Jean-Pierre Changeux and I have presented a theoretical model of the neuronal implementation of the log-Gaussian hypothesis (Dehaene and Changeux, 1993), later 
elaborated by others (Verguts and Fias, 2004; Verguts et al., 2005). Our model illustrates how approximate numerosity can be extracted from a retinotopic map through three successive stages: (1) retinotopic coding of object locations regardless of object identity and size; (2) representation of total activity by accumulation neurons which simply sum the activation on the object location map; (3) representation of each approximate numerosity by a distinct set of numerosity detector neurons, each tuned to a specific numerosity.

Because human and nonhuman animals can represent a large range of numbers (e.g. Cantlon and Brannon, 2006), we postulated a logarithmic spacing of neural thresholds, such that a decreasing number of neurons was allocated to increasingly larger numerosities (the alternative hypothesis of linear coding seemed implausible as it would imply that the vast majority of neurons encode large numerosities, although these numerosities are quite hard to discriminate). This principle of compressive coding was inspired by the known over-representation of species-relevant parameter ranges in sensory maps (e.g. fovea in retinotopic maps, hand and face in somatosensory maps, or even echo-locating frequencies in bat tonotopic maps). Simulations then showed that those neurons had approximately Gaussian tuning curves when plotted on a logarithmic axis—a neuronal implementation of the log-Gaussian hypothesis.

In the Dehaene-Changeux model, then, the firing rate of a numerosity detector neuron that responds preferentially to numerosity $p$, in response to a range of stimulus numerosities $n$, traces a bell-shaped curve which is Gaussian on a log scale and has a maximal firing peak at the location $p$. Mathematically, this tuning curve is given by:

$$
f(n, p)=\alpha G\left(\frac{\log (n)-\log (p)}{w^{\prime}}\right)=\alpha \frac{e^{-\frac{(\log (n)-\log (p))^{2}}{2 w^{\prime 2}}}}{\sqrt{2 \pi} w^{\prime}}
$$

In this equation, $G$ is the normal curve and $w^{\prime}$ is the neural Weber fraction which defines the degree of coarseness with which neurons encode numerosity. Note that $w^{\prime}$ can be quantitatively different from the psychologically defined internal Weber fraction $w$. This is because there is no simple relation between the single-neuron representation level (where tuning curves are characterized by parameter $w^{\prime}$ ), and the psychological representation level (where the precision of a subject's representation is characterized by parameter $w$ ). Intuitively, it is easy to envisage cases in which these parameters are dissociated. For instance, even if single neurons had a very coarse tuning curve (high $w^{\prime}$ ), it might still be possible to perform precise psychological judgment (low $w$ ) by averaging across an entire neural population. Conversely, even if some neurons had very precise codes (low $\left.w^{\prime}\right)$, it might not be possible for decision mechanisms to separate their signals from those of other less informative neurons, thus resulting in a psychological level of performance lower than the best performance theoretical achievable (high $w$ ).

The neurophysiological literature contains many discussions of the bridging laws that relate single-neuron coding to psychophysical representation, particularly in the welldocumented domain of movement perception (Shadlen et al., 1996; Parker and Newsome, 1998; Shadlen and Newsome, 1998). There is no consensus yet on the best 
formulation of these bridging laws. Their determination requires careful analysis of the trial-to-trial firing rate variability, number of neurons contributing to behavior, structure of correlations between them, and pooling rule used to combine their responses into a single summary value. Nevertheless, some basic results are available. A simple analysis indicates that, if a bank of neurons with Gaussian tuning is used to encode a certain magnitude, then the best way to estimate which value is represented by this population is to compute their population vector, which is the mean of the preferred values of each neuron, weighted by their current firing rate [mathematically, this is the best estimator in the maximum likelihood sense (Dayan and Abbott, 2001, pp. 106-108)]. According to this population-vector model, a set of log-Gaussian neurons predicts a log-Gaussian psychophysical internal scale-but not necessarily with the same quantitative variability parameters $w$ and $w^{\prime}$.

\section{Experimental evidence}

Behaviorally, the main prediction of the log-Gaussian model concerns the metric of similarity between the representations of two numerosities $n_{1}$ and $n_{2}$ (with $n_{1}<n_{2}$ ). The model predicts that the judged similarity between two numerosities should vary monotonically with the difference of their logarithms.

$$
\text { Similarity }\left(n_{1}, n_{2}\right)=S\left(\left|\log \left(n_{1}\right)-\log \left(n_{2}\right)\right|\right)=S(\log (r))
$$

where $S$ is monotonically decreasing and $r=n_{2} / n_{1}$ is the ratio of the two numbers.

Subjective similarity ratings conform to this rule (Shepard et al., 1975). When subjects rated the conceptual similarity of two numbers, regardless of whether they were denoted by dot patterns, digits or words, a logarithmic similarity scale was recovered by nonmetric multidimensional scaling.

At the neural level, Dehaene and Changeux's (1993) model predicts the existence of a hierarchy of several types of neurons: object maps, accumulation neurons, and numerosity detector neurons. At present, strong evidence exists only for the latter type of cells (Nieder et al., 2002; Nieder and Miller, 2003, 2004). Their properties conform in great detail to the proposed log-Gaussian model. Nieder and Miller trained macaque monkeys in a numerical match-to-sample task. On each trial, they attended to the numerosity of a sample set of visual dots and memorized it. After a delay, they were presented with a second numerosity and decided whether it was equal to the first. During both sample and delay periods, many neurons were tuned to a preferred numerosity, in the sense that they fired maximally to a given number of dots, and showed decreasing firing rates when the numerosity was smaller or larger than this preferred value. Collection of data from hundreds of trials led to a very precise characterization of each neuron's tuning curve. The hypothesis of a Gaussian curve on a linear axis (scalar variability) could be rejected. Rather, asymmetries in the tuning curves were compatible with the log-Gaussian hypothesis of a fixed-width Gaussian tuning curve once plotted as a function of $\log (n)$.

The numerosity-tuned neurons were initially found in prefrontal cortex, but later recordings in the depth of the intraparietal sulcus revealed another population of 
number neurons in, or close to, area VIP. The parietal cells differed from the prefrontal cells in two ways: they had a significantly faster latency, and they fired less strongly during the delay. Thus, the data are compatible with the hypothesis that numerosity is first computed and represented in intraparietal cortex, then transferred to prefrontal cortex for memory purposes. The object map and linear accumulation neurons postulated by the theory might then be tentatively associated with area LIP, which is retinotopic, monosynaptically connected to VIP, and thought to encode a saliency map of relevant object locations. Indeed very recently, neurons whose firing rate vary monotonically with number have been identified in area LIP (Roitman, Brannon and Platt, 2007).

The location where numerosity-sensitive neurons are found is a plausible homolog of the human intraparietal region found active during many mental arithmetic and numerical judgment tasks (Dehaene et al., 2003). Indeed its location in the depth of the macaque intraparietal sulcus could be roughly predicted from the finding of a homologous geometrical arrangement of surrounding sensorimotor regions in humans and macaques (Simon et al., 2002, 2004).

Following Nieder and Miller's finding, Manuela Piazza and I examined whether numerosity coding by log-Gaussian numerosity detectors could also be demonstrated in the human intraparietal sulcus (Piazza et al., 2004). Since we could not record from single human neurons, we took advantage of the functional magnetic resonance imaging (fMRI) adaptation method. While the subjects passively attended to the screen, we adapted them by repeatedly presenting, for several minutes, the same adaptation numerosity $n_{\text {hab }}$, which could be either 16 or 32 dots on different runs. We then presented occasional trials where the numerosity $n_{\mathrm{dev}}$ deviated by a variable amount from the reference value, up to twice smaller or twice larger. As in Nieder and Miller's study, stimuli were generated randomly by a Matlab program which provided precise control over non-numerical parameters (Dehaene et al., 2005). Based on past work, we expected repetition suppression, a decrease in the activity of the neurons coding for the adaptation numerosity. Using the above firing-rate function $f(n, p)$, we could predict mathematically the amount of activation expected to be elicited by a given deviant numerosity. Intuitively, this activation should reflect the combination of two Gaussians: one evoked by the adaptation numerosity, which created a Gaussian 'trough' in the neural population around the location of the adaptation value, and the second evoked by the deviant stimulus which is used to 'read out' the state of adaptation of the representation, and activates a Gaussian population of numerosity detectors which have been more or less adapted depending on their proximity to the adaptation value. Mathematically, the total activation which results from this combination is given by an operation called the convolution of the two Gaussians (see appendix of Piazza et al., 2004, for details). Thus, we expected the recovery from adaptation also to follow a Gaussian function of the difference of the logarithms of $n_{\text {hab }}$ and $n_{\text {dev }}$, but with a width larger than the neural tuning curve by a factor of $\sqrt{2}$ :

$$
I\left(n_{\mathrm{hab}}, n_{\mathrm{dev}}\right)=\lambda-\mu \frac{e^{-\frac{\left(\log \left(n_{\mathrm{hab}}\right)-\log \left(n_{\mathrm{dev}}\right)\right)^{2}}{4 w^{\prime 2}}}}{2 \sqrt{\pi} w^{\prime}}
$$


The fMRI data conformed in great detail to this model. In a whole-brain search, only the left and right intraparietal regions showed an fMRI response which depended on the amount of numerical deviancy. The shape of this response was tightly fitted by a Gaussian once plotted on the appropriate logarithmic scale. Furthermore, in log scale the Gaussian had a similar width for adaptation values 16 and 32, thus showing that Weber's law holds. In the absence of direct single-neuron recordings in humans, those data, replicated by others in adults and 4-year-olds (Cantlon et al., 2006; see also Temple and Posner, 1998), provide suggestive evidence that the same principle of log-Gaussian coding might be underlying numerosity perception in human adults, children, and nonhuman primates.

Recently, this method of numerosity adaptation has been extended to 2-3 month-old infants (V. Izard, G. Dehaene-Lambertz and S. Dehaene, unpublished data; V. Izard, PhD thesis, December 2005). We collected event-related potentials during adaptationdishabituation with numerosities 2 versus 3,4 versus 8 , or 4 versus 12 . Once babies were adapted to one of these numerosities, a right parietal negativity was evoked whenever the corresponding deviant was presented. Although lacking in precise localization, those results tentatively suggest that the parietal numerosity representation may be in place at a very early age in human development possibly with an early right hemisphere bias.

\section{Open issues}

A debated issue concerns whether behavioral and even neuroimaging or neurophysiological data may ever separate the log-Gaussian model from the linear scalar variability model. Randy Gallistel (personal communication) has repeatedly argued that they cannot. Behaviorally, indeed, I have demonstrated that both models predict an essential identical ratio dependence in a broad variety of judgments (S. Dehaene, unpublished work; see also below). At the neural level, Gallistel argues that a similar argument applies: the firing of the neurons can be considered as a sort of decision whose profile can be predicted from an underlying linear coding scheme with scalar variability. According to Gallistel, if each neuron were programmed as an ideal detector of numerosity on a linear continuum with scalar variability, it would show precisely the tuning curve asymmetries and ratio dependence that are thought to support the log-Gaussian model! If this argument is correct, then the models truly are inseparable, and are in fact mathematically equivalent. I would argue, however, that for fear of an infinite regress, it is not correct to postulate yet another, deeper level of linear representation on which the neurons act as optimal encoders. The presumption is that Nieder and Miller have recorded from what constitutes the brain's neuronal representation of number, and the log-Gaussian model appears to provide the most compact description of that code.

There may be other, less controversial ways to separate the models. The log-Gaussian model predicts a uniform distribution of preferred numerosities on a logarithmic scale, hence more neurons dedicated to small numerosities than to large ones. In Nieder and Miller's initial work, it was not possible to test this prediction, because only the numerosities 1-5 were tested. Nieder and Merten (2007) however extended this work to numerosities 1-30 and found that increasingly fewer neurons were tuned to larger numbers, compatible 
with the log-Gaussian model. It may not be entirely unrelated that, in all languages of the world, the frequency with which we name a given numerosity $n$ is a sharply decreasing function of $n$ (Dehaene and Mehler, 1992).

Against the log-Gaussian hypothesis, Brannon et al. (2001) have argued that, if numerosity were represented on a logarithmic internal scale, addition and subtraction operations would not be possible. Empirically, they showed that pigeons could be successfully trained to perform an approximate subtraction task. More generally, Gallistel and Gelman (1992) argued that a mental representation should be characterized by the type of operation it supports, and that competence for addition and subtraction provides incontrovertible evidence for a linear mental number line. In my opinion, however, this argument confounds the content of a representation with its form, what is being represented and how it is represented, or in Saussurian terms, the signified and the signifier. In the log-Gaussian model, what is being represented is a number $n$, although its internal representative is $q(n)=\log (n)$. It is equally absurd to state that when computing a subtraction $n_{1}-n_{2}$, we should then subtract the logarithms of $n_{1}$ and $n_{2}$, than to say that, because a computer encodes numbers in binary format, it should always compute $1+1=10$ ! In brain and computer alike, the rules of transformation of the representatives $q\left(n_{1}\right)$ and $q\left(n_{2}\right)$ should be stated so that the result of the internal operation is isomorphic to the desired arithmetic operation. In the case of the subtracting pigeons, I showed that, whether the numbers were coded by a linear or a logarithmic scheme, a simple neural network could easily pass the behavioral test that Brannon et al. took as diagnostic of a linear subtraction operation (Dehaene, 2001). The issue of calculation algorithms is discussed further below.

A separate open issue concerns whether the proposed 'number line' representation constitutes the sole semantic representation of number. There is evidence, at least in human infants, for a distinct system of representation of small sets of objects $(1,2$ or 3$)$, thought to be based on object or event files, and not subject to Weber's law (for review, see Feigenson et al., 2004). This system might be responsible for 'subitizing', the capacity to quickly and accurately name numerosities 1, 2 and 3 in adults. An alternative possibility, however, is that subitizing merely represents performance at the lower, most precise end of the number line continuum (Mandler and Shebo, 1982; Dehaene and Cohen, 1994; Cordes et al., 2001; Piazza et al., 2003). In animals, performance is quite often continuous over the whole range of numbers, with little or no evidence in favor of a distinct 'subitizing' system (Brannon and Terrace, 2000; Nieder et al., 2002; Nieder and Miller, 2003, 2004; Cantlon and Brannon, 2006; but see Hauser and Carey, 2003). Thus, whether a distinct system exists, what are its neural mechanisms, and how they might be modeled mathematically remain open issues.

\section{Numerosity discrimination, comparison and identification}

I now turn to the utilization of the numerosity representation in simple cognitive tasks. Based on the postulated log-Gaussian representation, and assuming a certain form for the decision system, can one reconstruct animal and human performance in simple tasks? 
I start with the prediction of performance (percentage correct) in various numerosity tasks, assuming that decision in this case is based on a single internal sample on the internal number line.

\section{Theory}

The theoretical principles applied here are identical to those used in signal detection theory to characterize psychophysical judgments. In turn, these principles can be derived from optimal Bayesian decision based on maximum likelihood or maximum a posteriori inference (Green and Swets, 1966; MacMillan and Creelman, 1991). In the most general terms, a behavioral experiment consists in presenting a set of numerical stimuli $S_{i}$, each of which is associated with one member of the set of responses $R_{j}$. On a given trial, the stimulus $S$ is represented by a noisy random variable $X$ on the internal continuum. The aim of the decision system is to select, among the set of possible responses $R_{j}$, the response $\mathrm{R}$ that has the greatest probability $P\left(R_{j} \mid \mathrm{x}\right)$ of being correct, given the state $x$ of the internal representation (the analysis can also be extended to the maximization of rewards associated with each response). Optimal responding can be achieved by finding the response $R$ that maximizes this probability $P\left(R_{j} \mid \mathrm{x}\right)$. By Bayes' rule,

$$
P\left(R_{j} \mid x\right)=P\left(x \mid R_{j}\right) P\left(R_{j}\right) / P(x)
$$

In this equation, only the first two terms vary with $R_{j}$. The first can be calculated: it consists in the mean of the Gaussian curves evoked by all the stimuli for which the correct response is $R_{j}$. The second term is often constant (all responses are equiprobable) or can be estimated over trials (possibly generating some bias).

The end result of this procedure is a family of optimal response curves $\left[g_{j}(x)=P\left(R_{j} \mid \mathrm{x}\right)\right]$ which specify, for every possible value of the internal representation $x$, what is the probability that the response $R_{j}$ is the correct one. ${ }^{2}$ The optimal strategy, when observing an internal representation $x$, consists in selecting the response $R_{j}$ for which $g_{j}(x)$ is the largest. This strategy defines a set of criteria that divide the internal number line into regions that should be responded to with different responses $R_{i}$. Once these criteria are set, it is easy to compute the probability of making a correct response to each stimulus $S_{i}$. One simply has to consider all of the possible internal encodings $x$ of this stimulus, and to examine for which fraction of them the above decision rule leads to the correct answer. Mathematically, this is given by the integral of the internal Gaussian representation over the interval of response criteria that are associated with the desired response to that stimulus. In the same way, the entire stimulus-response matrix which defines the probability of making any given response to any given stimulus can be computed.

2 In cases where a trial consists in the presence of two numerosities $n_{1}$ and $n_{2}$, those functions are defined over two internal variables $x_{1}$ and $x_{2}$ instead of one. Nevertheless the logic of finding which response has the highest probability of being correct remains the same. Furthermore, depending on the arrangement of the stimuli, one may frequently make the simplifying assumption that subjects base their decisions on a reduced variable such as the difference between $x_{1}$ and $x_{2}$. This approach has been adopted in what follows. 


\section{Application to experimental examples}

To make this presentation more concrete, we now consider several simple examples.

\section{Numerosity discrimination}

The theory and experiments for this task have been presented by Van Oeffelen and Vos (1982). Briefly, in a block of trials, the subject is presented with one of two numerosities (e.g. 15 or 18 dots) and has to decide which numerosity is presented. Thus, the stimulus set comprises only two numbers $n_{1}$ and $n_{2}$ (with $n_{1}<n_{2}$ ), and the response set is also limited to two responses: $R_{1}=$ ' $n_{1}$ ' and $R_{2}=$ ' $n_{2}$ '. Assuming equiprobable responses, the optimal response curves can be derived from the log-Gaussian representation curves according to:

$$
g_{j}(x)=P\left(R_{j} \mid x\right)=\frac{G\left(\frac{x-\log \left(n_{j}\right)}{w}\right)}{G\left(\frac{x-\log \left(n_{1}\right)}{w}\right)+G\left(\frac{x-\log \left(n_{2}\right)}{w}\right)}
$$

where $G$ is the normal curve. It is easy to see that $g_{2}(x)>g_{1}(x)$ if and only if $x>c$, where $c=\frac{1}{2}\left[\log \left(n_{1}\right)+\log \left(n_{2}\right)\right]=\log \left(\sqrt{n_{1} n_{2}}\right)$ is the response criterion. Hence, the optimal strategy, quite intuitively, consists in responding $R_{1}={ }^{\prime} n_{1}$ ' if the internal representation of the target falls closer to $\log \left(n_{1}\right)$ than to $\log \left(n_{2}\right)$, and to respond $R_{2}=$ ' $n_{2}$ ' otherwise. The predicted performance is thus

$$
P_{\text {correct }}\left(n_{1}, n_{2}\right)=\int_{-\infty}^{\log \left(\sqrt{n_{1} n_{2}}\right)} \frac{e^{-\frac{\left(x-\log \left[n_{1}\right]\right)^{2}}{2 w^{2}}}}{\sqrt{2 \pi} w} d x=\int_{-\infty}^{+\infty} \frac{e^{-\frac{\left(x-\frac{1}{2} \log [r]\right)^{2}}{2 w^{2}}}}{\sqrt{2 \pi} w} d x
$$

Note that the latter form makes clear the dependence of performance on the ratio of the two numbers, $r=n_{2} / n_{1}$ (one version of Weber's law). Van Oeffelen and Vos (1982) tested this equation against human numerosity discrimination data, and found it to be quite accurate.

Ratio-dependent performance has also been observed during numerosity discrimination in human infants (Lipton and Spelke, 2003). Although the data are probably not quantitative enough for a formal fit of our equations, the Weber fraction appears to decrease with age: 6-month-old babies fail to discriminate numerosities in a 3:2 ratio, while 9-month-old babies can (Lipton and Spelke, 2003).

\section{Numerosity comparison}

In the nonsymbolic number comparison task, on each trial subjects are presented with a single set of dots and have to determine if its numerosity is larger or smaller than some fixed reference $n_{\text {ref }}$. Thus, the set of stimuli can be large, but the set of responses is reduced to two responses: larger or smaller. An analysis of the optimal strategy, along the above lines, shows that there is a single criterion $c$ on the internal number line, and that subjects' response should be 'larger' if the internal representation exceeds this criterion, and 'smaller' otherwise. The optimal criterion $c$ generally coincides with, or is close to, 
the internal representation of $n_{\text {ref }}: c=\log \left(n_{\text {ref }}\right)$. For each target number, one can then predict the fraction of choices of the larger response as:

$$
P_{\text {larger }}\left(n, n_{\text {ref }}\right)=\int_{-c}^{+\infty} \frac{e^{-\frac{(x-\log [n])^{2}}{2 w^{2}}}}{\sqrt{2 \pi} w} d x=\int_{-\infty}^{+\infty} \frac{e^{-\frac{(x-\log [r])^{2}}{2 w^{2}}}}{\sqrt{2 \pi} w} d x
$$

Again, this equation makes apparent that performance should depend on the ratio of the stimulus and reference numbers, $r=n / n_{\text {ref }}$.

At least two successful tests of this equation have been published. First, Piazza et al. (2004) collected numerosity comparison judgments in adults. As shown in Figure 24.2, Equation 24.8 fitted the data very tightly. Ratio dependence was tested explicitly by testing two reference numbers, 16 and 32, and verifying that the slope of the psychophysical curves shifted by a factor of 2 on a linear scale (and was constant when plotted on a log scale). Second, Pica et al. (2004) used a slightly different version of the comparison task, where the subject sees two successive numerosities $n_{1}$ and $n_{2}$, and decides which is the larger. The theory is only slightly different here, and assumes that subjects respond ' $n_{1}>n_{2}$ ' if and only if the internal representatives are in the same order $x_{1}>x_{2}$. It is easy to obtain analytic expressions for error rates, both under the log-Gaussian and under the scalar variability hypotheses:

Log-Gaussian model: ${ }^{3}$

$$
P_{\text {correct }}\left(n_{1}, n_{2}\right)=\int_{0}^{+\infty} \frac{e^{-\frac{1}{2}\left(\frac{\left(x-\left(\log \left(n_{2}\right)-\log \left(n_{1}\right)\right)\right.}{\sqrt{2} w}\right)^{2}}}{\sqrt{2 \pi} \sqrt{2} w} d x=\int_{0}^{+\infty} \frac{e^{-\frac{1}{2}\left(\frac{x-\log (r)}{\sqrt{2} w}\right)^{2}}}{\sqrt{2 \pi} \sqrt{2} w} d x
$$

Linear, scalar variability model:

$$
P_{\text {correct }}\left(n_{1}, n_{2}\right)=\int_{0}^{+\infty} \frac{e^{-\frac{1}{2}\left(\frac{x-\left(n_{2}-n_{1}\right)}{w \sqrt{n_{1}^{2}+n_{2}^{2}}}\right)^{2}}}{\sqrt{2 \pi} w \sqrt{n_{1}^{2}+n_{2}^{2}}} d x=\int_{0}^{+\infty} \frac{e^{-\frac{1}{2}\left(\frac{x-(r-1)}{w \sqrt{1+r^{2}}}\right)^{2}}}{\sqrt{2 \pi} w \sqrt{1+r^{2}}} d x
$$

Both equations yield virtually identical predictions which, as made clear by the above equations, again depend solely on the ratio of the two numbers, $r=n_{2} / n_{1}$ (with $n_{1}<n_{2}$ ). Pica et al. (2004) collected data from children and adult, both in French subjects and in Munduruku Indians from the Amazon. In all cases, performance conformed to the above equations, thus suggesting that numerosity judgments belong to a core set of arithmetic knowledge that is available independently of language, culture and education.

3 Note that the sole difference with the equation characterizing performance in comparison with a fixed reference is a factor of 2 on the scaling of the parameter $w$. See MacMillan and Creelman (1991) for a cogent explanation of such subtle differences between psychophysical paradigms, particularly what they call two-choice tasks (where two stimuli $n_{1}$ and $n_{2}$ are presented) versus reminder tasks (where a single stimulus $n$ is compared to a fixed reference). 


\section{Same-different judgments}

Another simple task consists in asking subjects whether two numbers are the same or different. Piazza et al. (2004) used this task with a fixed reference. Subjects were presented on each trial with a single numerosity $n$ and had to decide whether this numerosity was identical to, or different from, a fixed reference $n_{\text {ref }}$ (this reference was fixed for an entire block, and subjects were reminded of it on each trial). If the different target numerosities are symmetrically distributed around the habituation numerosity on a logarithmic scale (which was the case in Piazza et al.'s stimulus set), then the subject's optimal criterion consists in responding 'same' whenever the internal representation of the stimulus numerosity $n, x$, falls within a symmetrical decision interval centered around the representation of the reference number. Thus, subjects should respond 'same' if and only if $x$ belongs to $\left[\log \left(n_{\text {ref }}\right)-\delta, \log \left(n_{\text {ref }}\right)+\delta\right]$, where $\delta$ defines the width of the criterial region. Given this response strategy, the probability of responding 'same' to a stimulus numerosity $n$ is given by:

$$
P_{\text {same }}\left(n, n_{\text {ref }}\right)=\int_{\log \left(n_{\text {same }}\right)-\delta}^{\log \left(n_{\text {same }}\right)+\delta} \frac{e^{-\frac{(x-\log (n))^{2}}{2 w^{2}}}}{\sqrt{2 \pi} w} d x=\int_{-\delta}^{\delta} \frac{e^{-\frac{(x-\log (r))^{2}}{2 w^{2}}}}{\sqrt{2 \pi} w} d x
$$

Again, performance should depend on the ratio of the stimulus and reference numbers, $r=n / n_{\text {ref }}$. As shown in Figure 24.2, an excellent fit with experimental data was observed, and ratio dependence was verified with two reference numbers, 16 and 32 .

A related task was used by Nieder and Miller (2003) in macaque monkeys. As described earlier, on each trial, subjects were first presented with a sample number $n_{1}$, then after some delay with a second number $n_{2}$. They had to decide whether the first number matched the second, or differed from it. As shown in Figure 24.1, performance averaged across a very large number of trials was a remarkably regular function of the difference of logarithms of the two numbers (or, equivalently, of their log ratio), which Nieder and Miller (2003) showed to be well captured by a Gaussian curve.

According to the theory, a simplified though frequently close to optimal strategy for this task is to respond 'same' whenever the difference between the internal representatives $x_{1}$ and $x_{2}$ falls below a certain criterion $\delta$. The probability of responding 'same' is then given by:

$$
P_{\text {same }}\left(n_{1}, n_{2}\right)=\int_{-\delta}^{-\delta} \frac{e^{-\frac{1}{2}\left(\frac{x-\left(\log \left(n_{2}\right)-\log \left(n_{1}\right)\right)}{\sqrt{2} w}\right)^{2}}}{\sqrt{2 \pi} \sqrt{2} w} d x=\int_{-\delta}^{\delta} \frac{e^{-\frac{1}{2}\left(\frac{x-\log (r)}{\sqrt{2} w}\right)^{2}}}{\sqrt{2 \pi} \sqrt{2} w} d x
$$

where $r=n_{2} / n_{1}$ (with $n_{1}<n_{2}$ ). Note that this equation, the integral of a Gaussian over a given interval, departs from the simple Gaussian used by Nieder and Miller (2003) to fit their data. Nevertheless, I have verified that the two fits are essentially indistinguishable and that the present equation thus provides an excellent account of the performance of Nieder and Miller's monkeys in numerical match-to-sample tasks. 
A

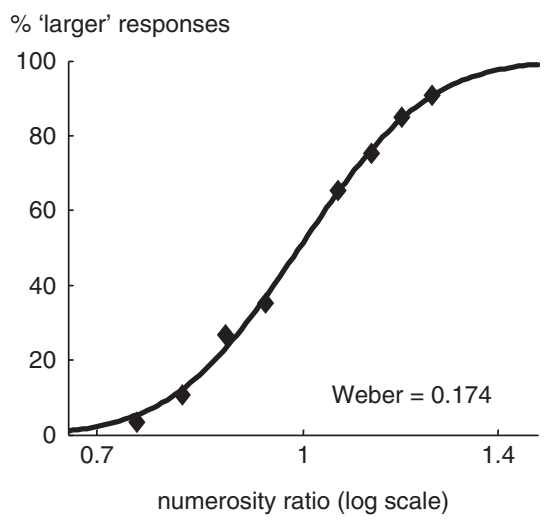

B Same-different judgment

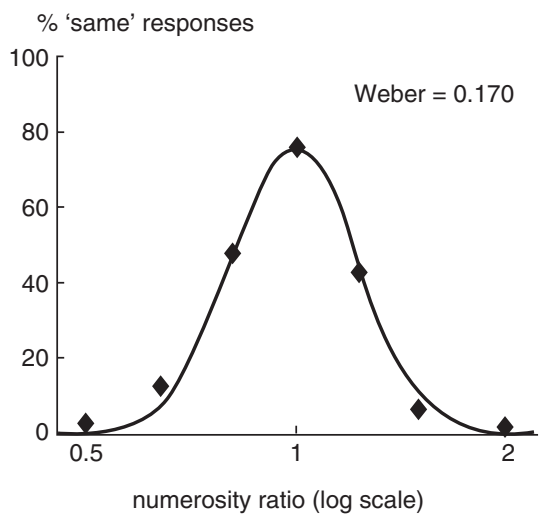

$\mathrm{C}$ Numerosity match-to-sample

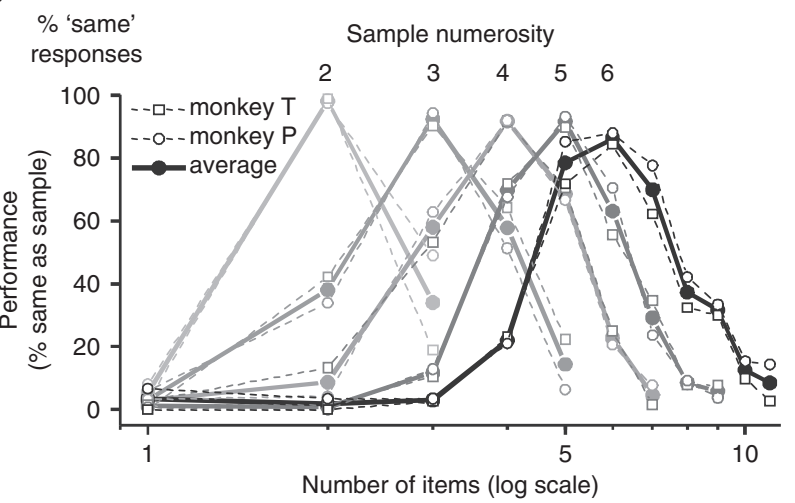

Figure 24.2 Human and macaque monkey performance in simple numerosity tasks is well-captured by the proposed psychophysical model. (A) Humans, larger-smaller comparison of large numerosities to a fixed reference (16 or 32). (B) Humans, same-difference judgment of large numerosities with a fixed reference (16 or 32). Both data sets are redrawn from Piazza et al. (2004). The dots are experimental data points, and the curve is the best fit by equations described in the text. The dependence of performance on the log numerosity ratio is evident. (C) Data from Nieder et al. (2002) for same-different judgment. The monkeys decide whether a sample numerosity (numeral appearing above each curve) matches a subsequently presented numerosity (abscissa, log scale). The curves appear as shifted versions of the curve in (B) again indicating that performance depends solely on log ratio.

\section{Numerosity labeling}

A final task consists in asking the subject to label numerosities using a set of verbal or nonverbal labels. For instance, one may ask human subjects to label sets of dots ranging from 10 to 100 with round numbers such as the decade names 'ten' to 'ninety' (Izard, 2005). Chimpanzees have also been trained to label numerosities using the Arabic digits 1-9 (Matsuzawa, 1985; Tomonaga and Matsuzawa, 2002). In humans, this task can be complicated by the use of slow but exact counting strategies, which fall beyond the scope 
of the present theory. In animals, however, and perhaps also in human cultures with few number words and no overt counting system, the proposal is that subjects do not count serially, but merely apply symbolic labels to their mental representations of approximate numerosity (Dehaene and Mehler, 1992; Tomonaga and Matsuzawa, 2002; Gordon, 2004; Pica et al., 2004).

The theory for such numerosity-labeling tasks, developed by Izard (2005) assumes that, for each target numerosity $n$, subjects generate an internal representation $X$ of the target numerosity, thus a Gaussian random variable centered on $\log (n)$, and respond with the verbal label $r$ whose canonical representation on the number $\operatorname{line} \log (r)$ falls closest to $\log (n)$. This strategy implies that the number line continuum is divided into distinct response domains according to a set of response criteria forming a response grid (Figure 24.3A). The response domain corresponding to response $r_{1}$ is separated from the domain corresponding to the next response $r_{2}$ by response criterion

$$
c_{+}\left(r_{1}\right)=c_{-}\left(r_{2}\right)=\log \left(\sqrt{r_{1} r_{2}}\right)
$$

A

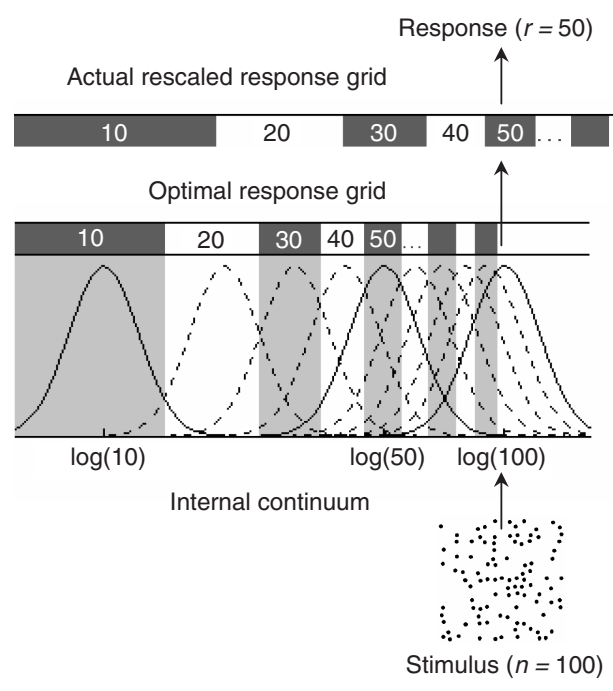

B
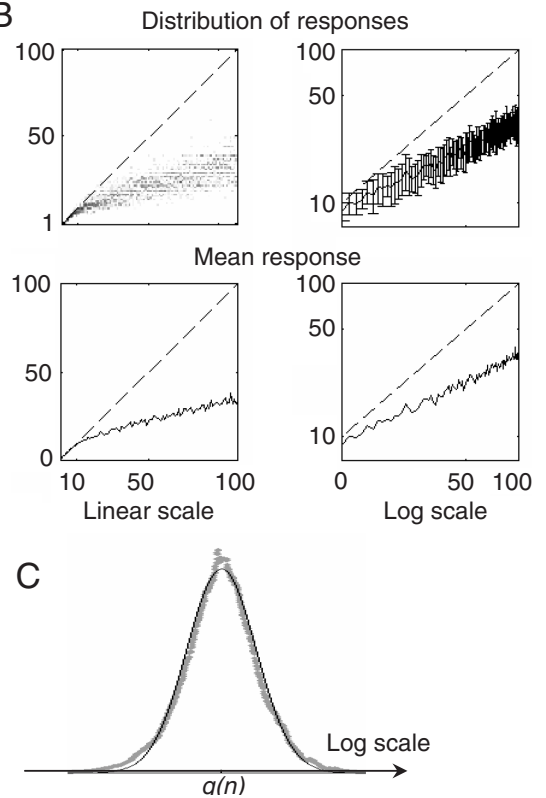

Figure 24.3 (A) Model of approximate numerosity naming (Izard, 2006). The input numerosity, once coded on the logarithmic number line, is categorized according to a response grid which is an affine rescaled version of the optimal logarithmically scaled grid. (B) Numerosity-naming responses of a representative subject, showing power-law responding with Weberian variability on a linear scale (left), and linear performance with fixed variability on a log-log scale (right). (C) Reconstructed distribution of the random variable leading to response choice [on a log scale, after centering on the modal value $q(n)$ and averaging across all targets and subjects]. The observed distribution tightly fits the predicted Gaussian. 
A complication is that subjects' responses are often poorly calibrated. For instance it is quite common for subjects to respond 'fifty' to a set of 100 or 200 dots (Minturn and Reese, 1951; Krueger, 1982). Véronique Izard showed that a simple assumption could still capture the subject's behavior. The assumption is that miscalibration is due to an affine scaling of the entire response grid. That is, instead of applying the optimal response criteria defined above, subjects actually use a linearly scaled grid of response criteria

$$
c_{+}^{\prime}\left(r_{1}\right)=a c_{-}\left(r_{1}\right)+b=a \log \left(\sqrt{r_{1} r_{2}}\right)+b
$$

where $a$ is a stretch parameter and $b$ a shift parameter, both of which typically differ from their optimal values (respectively 1 and 0 ).

Given this theory of the response selection process, the model predicts the frequency with which response $r$ will be selected in response to numerosity $n$ :

$$
P(n, r)=\int_{a c_{-}(r)+b}^{a c_{+}(r)+b} \frac{e^{-\frac{(x-\log (n))^{2}}{2 w^{2}}}}{\sqrt{2 \pi} w} d x
$$

In principle, this equation allows computation of any aspect of the subject's responses to any numerosity. In practice, it is difficult to obtain formal mathematical results. However, using plausible approximations, Izard (2005) was able to compute the subject's mean response to a given numerosity $n$. Interestingly, the response increases nonlinearly, as a power-law of the true numerosity $n$ :

$$
\overline{r(n)}=n^{\frac{1}{a}} e^{\frac{w^{2}}{2 a^{2}}-\frac{b}{a}}
$$

Izard (2005) showed that the above model provided a remarkably good fit to human subjects' numerosity naming data in the range 10-100. All subjects were initially miscalibrated and severely underestimated numerosity. In those cases, the predicted power-law relation was observed (see example in Figure 24.3B). A single example of a numerosityname pairing was sufficient to recalibrate them to a quasi-linear relation. This recalibration process was well captured by a change in the parameters $a$ and $b$. Furthermore, variability in the subjects' responses increased with stimulus numerosity on a linear scale, but became constant and with a linear stimulus-response relation once the data were plotted on a log-log scale (Figure 24.3B). Because a very large number of responses was collected for each subject, the data allowed for a reconstruction of the distribution of the internal random variable leading to response selection. On a logarithmic axis, this distribution traced an almost perfect Gaussian curve (Figure 24.3C).

In spite of these successes in modeling numerosity naming data, there are at least two directions where the theory will require extension. First, the theory assumes nonoverlapping response domains: each portion of the number line can be referred to by a single symbolic label. In natural speech, however, there are competing words or phrases for each numerosity. For instance the same quantity 13 can be truthfully named with variable degrees of precision as 'thirteen', 'a dozen' or 'ten-fifteen'. Dehaene and Mehler (1992) have proposed that each numeral has a defined response range, which is larger for round 
numbers such as 'fifteen' than for other numbers such as 'thirteen' (see also Pollmann and Jansen, 1996). Exactly how number word selection should be modeled in the presence of such competing responses and variable context remains to be determined.

A second area open to further research concerns tasks in which, instead of using a small set of discrete labels, the subject labels numerosity with a continuous or quasicontinuous response, for instance by tapping a key approximately as many times as the numerosity that was presented (Mechner, 1958; Whalen et al., 1999; Cordes et al., 2001). Such tasks probably do not involve the setting of a response grid, but rather a continuous monitoring of the numerosity being produced and a simple decision rule for stopping when that numerosity is thought to match or exceed the memorized one-a process which will not be further discussed here (but see for example Gibbon, 1977; Gibbon and Fairhurst, 1994).

\section{Numerical response times}

We now turn to the mental chronometry of arithmetic tasks. In many of the above tasks, response times vary systematically in parallel with the percentage of correct responses. Can arithmetic decision times also be accounted for by a simple mathematical model?

\section{Theory}

A simple idea concerning decisions under uncertainty was first introduced by Alan Turing in a cryptographic context, and a few years later by the statistician Abraham Wald (1947). In their view, the reason why decisions take a variable time which depends on the quality of the evidence, is because evidence must be accumulated until a pre-defined level of statistical certainty is achieved. The idea was imported into psychology by Stone (1960) and Laming (1968), then extended by Link (1975, 1990, 1992), Ratcliff (1988), Ratcliff and Rouder, 1998), and many others (e.g. Schwarz, 2001; Page et al., 2004; Sigman and Dehaene, 2005). It plays a key role in modern models of decision making and response time distributions (for accessible syntheses, see Gold and Shadlen, 2001, 2002; Usher and McClelland, 2001; Smith and Ratcliff, 2004).

Here I shall explain this theory only in the case of a simple two-alternative decision (e.g. larger-smaller comparison; see Figure 24.1). Assume that the subject is presented with a target numerosity $n$, but instead of a single representative $x$, now has at its disposal a time series of independent samples $x_{t}$ on the internal number line. According to Bayes' rule, each new sample allows updating of the posterior probability that the response $R_{j}$ is the correct one, given all of the previous samples:

$$
P\left(R_{j} \mid x_{1 . . t+1}\right)=P\left(x_{t+1} \mid R_{j}\right) P\left(R_{j} \mid x_{1 \ldots t}\right) / P\left(x_{t+1}\right)
$$

When only two responses are allowed, the mathematics can be simplified because a single quantity, the log-likelihood ratio (LLR), suffices to track how each additional sample changes the decision probabilities. This quantity is defined by the following equation:

$$
\operatorname{LLR}(t)=\log \left(\frac{P\left(R_{2} \mid x_{1 . . t}\right)}{P\left(R_{1} \mid x_{1 . . t}\right)}\right)
$$


Intuitively, the $L L R$ measures the relative amount of support for response 2 over response 1 . It is positive if the majority of the data supports response 2, and negative if it supports response 1. By Bayes' rule, one has

$$
\operatorname{LLR}(t+1)=\operatorname{LLR}(t)+\log \left(\frac{P\left(x_{t+1} \mid R_{2}\right)}{P\left(x_{t+1} \mid R_{1}\right)}\right)=\sum_{i=1}^{t+1} \log \left(\frac{P\left(x_{i} \mid R_{2}\right)}{P\left(x_{i} \mid R_{1}\right)}\right)+\log \left(\frac{P\left(R_{2}\right)}{P\left(R_{1}\right)}\right)
$$

This formula indicates that, by simple summation, one can add up the contributions of each random sample towards the decision (including a possible initial bias for one of the two responses). The successive bits of information contributed by each new sample should be added to produce an internal random walk. As time passes, the accumulated $L L R$ will vary somewhat randomly up and down. However, if there is a consistent signal in the internal samples, it should progressively drift towards either positive or negative values. An optimal decision consists in waiting until the LLR has reached one of two fixed bounds $\pm \theta$, and then responding with response $R_{2}$ if LLR is positive, and with response $R_{1}$ if LLR is negative.

The value of $\theta$, which is set by the subject, specifies the desired error rate. $\theta$ also determines how the subject deals with the speed-accuracy trade-off. Setting $\theta$ to a low value means that the decision threshold will be reached quickly, but with many errors (because internal noise will often lead the internal random walk to the wrong decision bound). Setting $\theta$ to a higher value means that decisions will be slower, but more accurate. A theory for the optimal choice of parameter $\theta$, depending on the rewarding scheme and inter-trial interval, has been presented (Bogacz et al., 2006).

\section{Discrimination task}

Although the formula that gives the increment to the LLR as a function of the observed internal sample $x_{i}$ is complex, it becomes simpler in some particular cases such as the above-discussed numerosity discrimination task, where one must simply decide which of two possible numerosities $n_{1}$ or $n_{2}$ was presented. In this case, the formula becomes:

$$
\operatorname{LLR}(t+1)=\frac{\log (r)}{w^{2}} \sum_{i=1}^{t+1}\left(x_{i}-\log \sqrt{n_{1} n_{2}}\right)+\log \left(\frac{P\left(R_{2}\right)}{P\left(R_{1}\right)}\right)
$$

Since the samples $x_{i}$ are Gaussian, this equation indicates that the random walk consists in a sum of Gaussian steps proportional to $x_{i}-c$, where $c=\log \left(\sqrt{n_{1} n_{2}}\right)$. Quite intuitively, those steps are positive if the sample $x_{i}$ is above the mid-point between $\log \left(n_{1}\right)$ and $\log \left(n_{2}\right)$, and negative otherwise. The mean and standard deviation of the step size are:

$$
\mu= \pm \frac{\log (r)^{2}}{2 w^{2}} \text { and } \sigma=\frac{\log (r)}{w}
$$

Once again, they indicate that all aspects of performance (errors, mean RT, RT distribution) should depend solely on the log ratio $r$ of the two numerosities $n_{1}$ and $n_{2}$. 


\section{Other tasks}

In most other tasks, the mathematics becomes more complicated. According to the normative theory of optimal decision making outlined above, the step size should be

$$
\log \left(\frac{P\left(x \mid R_{2}\right)}{P\left(x \mid R_{1}\right)}\right)
$$

This function of $x$ is usually rather complicated and need not be linear or even monotonic with $x$. Thus, the random-walk steps may have an unusual, mathematically intractable distribution. Nevertheless, in many cases the Gaussian random walk can be used as an analytically tractable approximation. It is also possible that the neural systems for decision making rely on such a Gaussian approximation because it is easier to compute neurally (Gold and Shadlen, 2001). Thus, in the literature, it is frequently assumed that each decision is based on a Gaussian random walk defined, for a given target numerosity $n$, by parameters $\mu$ and $\sigma$ specifying the mean and standard deviation of the steps per unit of time. If needed, one may compare those analytic calculations with simulations of many random walks that behave according to the exact $L L R$ equations.

\section{Predictions of the model}

In the Gaussian random-walk model, the decision time is defined as the first point at which the random walk crosses one of two absorbing barriers $+\theta$ and $-\theta$. Determining the distribution of decision time is a mathematically well-defined and physically wellknown diffusion problem, for which much is known (see e.g. Wald, 1947; Link, 1992). Here I consider only the simplest case where there is no initial bias for either response.

First, performance and error rates can be computed. A generic formula for response rates (valid also for non-Gaussian steps) is:

$$
P_{R=R_{2}}=\frac{1}{1+e^{-\lambda \theta}}
$$

where $\lambda$ depends on the distribution of the random walk steps

$\left(\lambda=\frac{2 \mu}{\sigma^{2}}\right.$ for Gaussian steps $)$.

This equation shows that, in the random walk model, the dependence of errors on mean step size $\mu$ (hence on distance in a number comparison task, for instance) is a sigmoid function. Remember that, in the classical signal detection theory, the predicted function is the integral of a Gaussian. Although those functions differ in theory, they are sufficiently similar as to be empirically indistinguishable. However, the random walk model presents the advantage of capturing the well-known observation of a speedaccuracy trade-off: as shown by Equation 24.22, performance is not fixed, but increases in a predictable manner with $\theta$, the response threshold.

The mean response times can also be predicted as:

$$
R T=T_{0}+\frac{\theta}{\mu}\left(2 P_{R=R_{2}}-1\right)
$$


where $T_{0}$ is a constant additional time corresponding to the total duration of nondecision processes (e.g. perceptual and motor components). Note that $R T$ decreases as an inverse function of step size, a function which is shallower than the above error curve. The sigmoid shape of errors and inverse shape of RTs as a function of log ratio constitute clear predictions of the random-walk model.

Finally, the variance and even the distribution of RTs can be calculated analytically (Smith and Ratcliff, 2004; Wagenmakers et al., 2006). There is an exact formula for the probability density function of RT, but it is quite complex. A simpler formula can be obtained if one considers, as a first approximation, the problem of a random walk process hitting a single barrier (thus neglecting the effect of errors). Obviously this will be a good approximation only if the task and subject threshold afford relative error-free performance. In this case, the probability density function of RT is

$$
p(R T \in[t, t+d t])=\frac{\theta}{\sigma \sqrt{2 \pi\left(t-T_{0}\right)^{3}}} e^{-\frac{\left(\theta-\mu\left(t-T_{0}\right)\right)^{2}}{2 \sigma^{2}\left(t-T_{0}\right)}} d t
$$

A problem with the random-walk model, known at least since Laming (1968), is that it predicts an identical distribution of correct and error RTs. This problem, however, has been corrected in slightly more complicated versions of the model that assume trial-totrial variability in the starting point of the random walk, the decision threshold, and/or the mean step size (Ratcliff and Rouder, 1998; Smith and Ratcliff, 2004). Naturally, those models have many additional free parameters.

\section{Model identification}

A specific difficulty with random-walk models is that their complexity often precludes easy identification of their parameters based on the available response time data. In the simplest model considered here, the minimum parameters that need to be fitted to each stimulus condition are: $T_{0}$ (nondecision time), $\theta$ (decision threshold), and $\mu$ (mean step size). ${ }^{4}$ Here I consider two simple and effective strategies (for more sophisticated approaches, see e.g. Ratcliff and Tuerlinckx, 2002). ${ }^{5}$

\section{Strategy 1}

This strategy, which I derived from equations in Link (1992), requires only the mean RT and mean error rate in each cell of the experimental design (e.g. for each target number $n$ ).

4 The standard deviation of step size, $\sigma$, is not independent of the other parameters once the time unit is fixed. It can thus be arbitrarily fixed (here, I took $\sigma=1$ ).

5 Both strategies will fail for cells of the experimental design which contain $0 \%$ or $100 \%$ errors. In such cases, it is possible to regularize the data by adding $1 / 2$ to the count of error trials. 
From Equations 24.22 and 24.23, we first derive a simple relation between mean RT and errors:

$$
R T(n)=T_{0}+\frac{4 \theta^{2}}{\sigma^{2}} \frac{P_{R=R_{2}}(n)-\frac{1}{2}}{\log \frac{P_{R=R_{2}}(n)}{1-P_{R=R_{2}}(n)}}
$$

This equation predicts that, across a range of stimulus conditions, there should be a linear relation between mean RT and a transformed function of the error rates. Thus, a first test of the random-walk model consists in checking, by linear regression, if this linear relation holds. If it does, then the intercept and the slope of the linear regression will provide estimates of $T_{0}$ and $\theta$ (assuming a fixed $\sigma$ ). Once these two parameters have been inferred, using Equation $24.23 \mu$ can be estimated for each target $n$ as

$$
\mu(n)=\frac{\theta\left(2 P_{R=R_{2}}(n)-1\right)}{R T(n)-T_{0}}
$$

The advantage of this procedure is that it includes an internal check of the validity of the random-walk model. The disadvantages include the assumption that $T_{0}$ and $\theta$ are identical across conditions, and that there is no response bias. The quality of the estimation is also highly dependent on the quality of the RT-error regression. If the data include errors due to another source, for instance guessing or inattention, then $T_{0}$ will be overestimated. For blocks of numerical comparison with a fixed notation, however, the assumption of fixed $T_{0}$ and $\theta$ is reasonable (the assumption is that perceptual and motor time are identical for all numbers, and that subjects set their criterion prior to seeing the target number). As we shall see, under these conditions the procedure recovers highly stable estimations of $\mu$.

\section{Strategy 2. Wagenmakers's EZ-diffusion model}

This strategy is described in detail in (Wagenmakers et al., 2006), so the equations will not be reproduced here. The strategy requires measurement of the mean RT, the variance of RT, and the mean error rate in each cell of the experimental design. From those three measures, an explicit formula recovers the parameters $\mu, T_{0}$, and $\theta$. Thus, an advantage of the EZ model is that it allows independent computation of all three parameters of the random-walk model separately for each cell of the experimental design. These parameters can then be submitted to an analysis of variance. Thus, unlike in strategy 1 , one can explicitly test whether $T_{0}$ and $\theta$ remain fixed across experimental conditions. On the negative side, the strategy assumes that there is no response bias (see Wagenmakers, 2006, for more complicated alternatives) and is somewhat unstable numerically. 


\section{Experimental evidence}

\section{Number comparison}

Number comparison is the numerical task that has received the most extensive treatment by the random-walk model (Link, 1975, 1990, 1992). I have verified that the model accounts for the performance and RTs in the data sets at my disposal (e.g. Dehaene, 1989; Dehaene et al., 1990, 1998; Piazza et al., 2004). Here I shall consider only a particularly dense set of data kindly provided to me by Cantlon and Brannon (2006), and which allows for a thorough test of the validity of the random-walk model in two species. In Cantlon and Brannon's experiment, two monkeys and 11 human subjects were presented with arrays of dots ranging from two to 30 dots. They had to select the smaller array or, in other blocks, the larger array. Here I have averaged across those two types of blocks.

Figure 24.4 shows the data and fits of the random-walk model obtained using strategy 1 . The top graphs indicate that, in both species, the variations of RT and error rate with the distance between the compared numerosities (as measured by the logarithm of their ratio) are well captured by the functions predicted by the model. I verified that a much worse prediction is obtained if the numerical distance is measured by the difference between the two numerosities, rather than by the difference of their logarithms.

Note that the distance effect is steeper on error rates than on mean RTs, as predicted. In each species, the bottom left graph examines the linear relation between RT and transformed error rate predicted by Link $(1975,1992)$. This relation is verified in both humans and monkeys, and allows for an estimation of $T_{0}$ and $\theta$. Interestingly, the response threshold $\theta$ is very similar across species (monkeys: 16.3; humans: 18.0), but the nondecision time appears slower in humans (monkeys: $342 \mathrm{~ms}$; humans: $462 \mathrm{~ms}$ ).

Most importantly, the mean step size of the random walk is an almost perfectly linear function of the log ratio of the two numbers (bottom right graphs in Figure 24.2). This linear function has a null intercept, i.e. the subjects appear not to be able to accumulate any information as the difference between the two numerosities becomes very small. The entire curve fits with the theory, which states that subjects accumulate a stochastic signal proportional to the difference of the log-Gaussian numerosity estimates provided by the two sets of dots. The slope of that function estimates, for a given numerical difference, the quantity of information that is accumulated per unit of time. Interestingly, this quantity, which plays a role similar to the internal Weber fraction in the simpler signal detection model, is somewhat smaller in monkeys (0.66) than in humans (0.99). This finding is in agreement with Cantlon and Brannon's conclusion that monkeys are somewhat less sensitive to numerosity information than (educated) humans.

\section{Neuronal modeling}

For a long time, the random-walk model was a purely psychological tool, and it was unclear whether it bore any relationship to actual neural network mechanisms. Recently, however, experiments and models on the neural mechanisms of decision making have flourished. Gold and Shadlen $(2001,2002)$ describe how neuronal populations might compute quantities relevant to the random-walk model. Furthermore, Mazurek et al. (2003) 

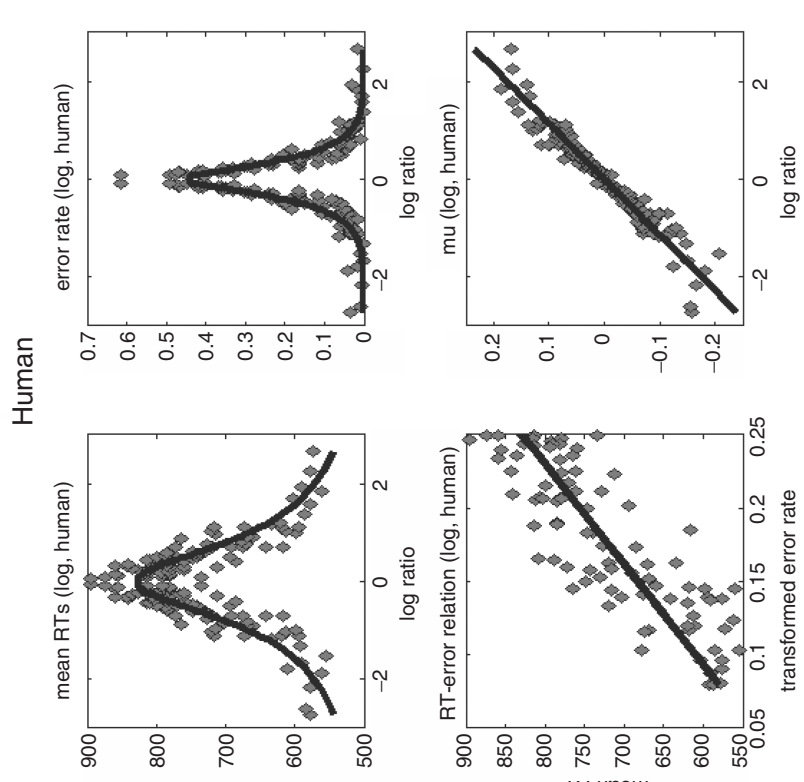

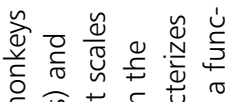

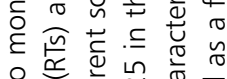

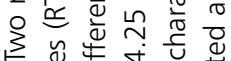

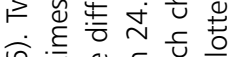

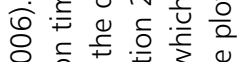

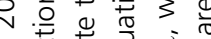

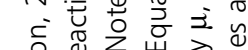

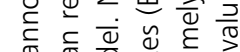

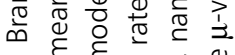

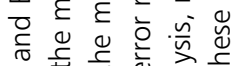

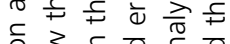

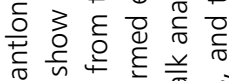
s.

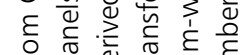

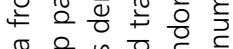
苟

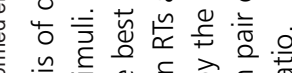

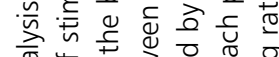

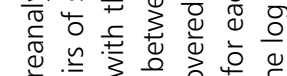

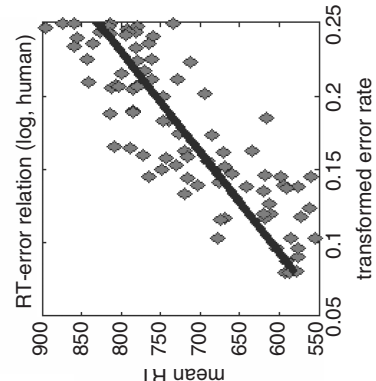

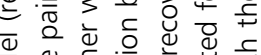
ब 它

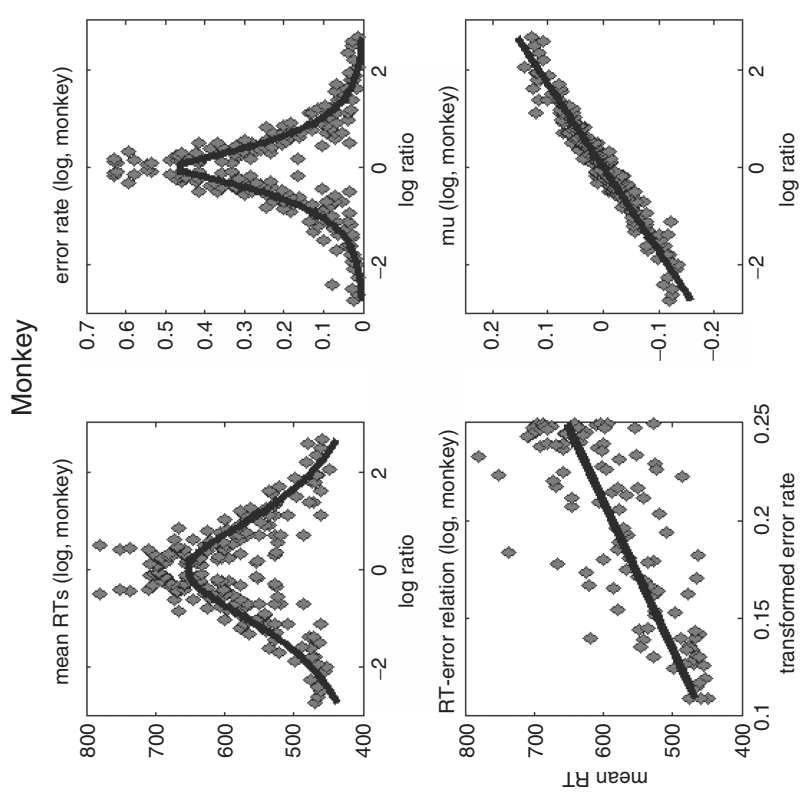
ये w

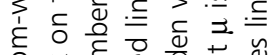

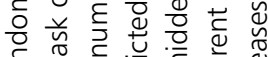

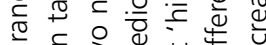
๘

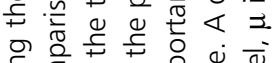
जह है एँ

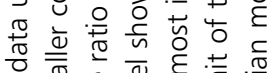
ᄃ

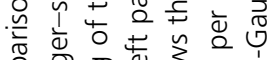

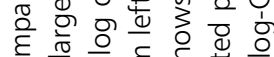

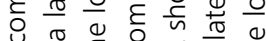

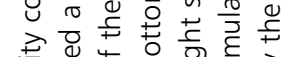

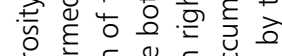
๘ँ

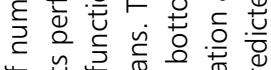

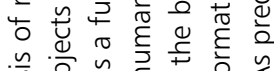

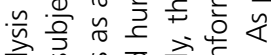

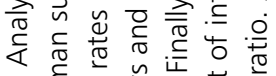

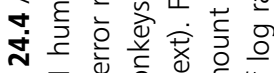
든

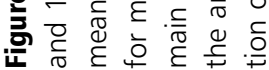


have proposed an explicit simulation model (see also Usher and McClelland, 2001), and Wong and Wang (2006) have described a neuro-realistic implementation of an accumulationlike neural decision process. Here, I briefly review these contributions.

Gold and Shadlen first note that the LLR, or a quantity monotonically related to it, can be quite easily computed as the difference of activity of two populations of neurons: those voting in favor of response $R_{1}$, and those voting in favor of response $R_{2}$. Thus, in the case of numerical tasks, a decision can be taken by selecting two relevant pools of numerosity-detecting neurons (e.g. those coding respectively for numbers smaller and larger than some reference), and computing the difference of their mean firing rates. The latter differencing operation can be accomplished either by a balance of excitatory and inhibitory feedforward connections, or by lateral competition between two competing populations of cells.

A second step consists in accumulating those difference signals. Mazurek et al. (2003) have shown how this can be accomplished by populations of decision neurons with a time delay and a self-connection. Intuitively, using those self-connections, the decision neurons can feed back onto themselves the neural activation that they received in the past, and hence maintain an accumulated record of the total amount. Wong and Wang (2006) have presented a realistic neuronal model of this process (see also Usher and McClelland, 2001; Machens et al., 2005). Wong and Wang consider pools of selective and nonselective excitatory neurons connected to a pool of inhibitory inter-neurons. They explicitly calculate the mean field dynamics of this system in the presence of an input signal favoring one or the other populations. The bifurcation diagram shows how this dynamical system approximates the accumulator needed in the random-walk theory: the accumulated evidence progressively pushes the activity of the two competing neuronal pools away from a saddle point and towards one of two attractors corresponding to the two possible decisions, in a time directly related to the amount of input evidence. ${ }^{6}$

Thus, two key components of the random-walk model (LLR formation and accumulator mechanism) have received a plausible neuronal implementation. Still, it should be noted that there is currently no comprehensive model of an entire decision task at the neuronal level. Such a model would require specifying: (1) how task instructions lead to the selection of the appropriate pools of neurons; (2) how these neurons become transitorily linked to the appropriate decision units, with the appropriate excitatory or inhibitory weights; (3) how the decision threshold, leading to motor response initiation, is implemented. A model of tactile frequency comparison, which comes close to achieving these goals, has been recently presented by Machens et al. (2005; see also Lo and Wang, 2006).

\section{Neurobiological and neuroimaging evidence}

The plausibility of the random-walk model of simple response decisions was greatly strengthened when Michael Shadlen and colleagues discovered neuronal signals that

6 Interestingly, in this neuronal model the evidence accrual process deviates from the neutral point at an exponential rate, a possibility that has rarely been considered in behavioral models, but may fit response time data better (see Page et al., 2004). 
appeared as plausible neural correlates of a stochastic accumulation process (Kim and Shadlen, 1999; Gold and Shadlen, 2001, 2002). Here I mention these data only briefly, because in spite of their intrinsic interest, they do not directly concern numerical tasks. During a motion judgment task, neurons whose firing appeared to constitute a plausible correlate of evidence accumulation were identified, first in prefrontal cortex (Kim and Shadlen, 1999), then in area LIP (Shadlen and Newsome, 2001) and other regions such as superior colliculus. The cells began deviating from baseline firing rates at a fixed latency of about $200 \mathrm{~ms}$ after the stimulus, and showed, on average, steadily increasing firing rates with a slope proportional to the amount of sensory evidence (here, the proportion of coherent motion in the display). Finally, they predicted the response of the animal, which appeared to be emitted once the neurons reached a fixed level of firing. All of these data have been captured in detail by the above-described neuronal models (Mazurek et al., 2003; see also Smith and Ratcliff, 2004; Wong and Wang, 2006; Lo and Wang, 2006).

Other tasks have yielded similar data. In particular, Ranulfo Romo's team has characterized neuronal firing in many cortical areas during a tactile frequency comparison task (Romo and Salinas, 2003). Romo et al. have identified neurons, particularly in prefrontal cortex, whose activity initially increases with the evidence accrued from the first stimulus; they then subtract the evidence accrued from the second stimulus before converging to a decision as to which of these two quantities is the largest. This process seems to be highly similar to that postulated for numerical comparison, although for unknown reasons tactile frequency appears to be encoded by the monotonically increasing or decreasing firing of neurons rather than by Gaussian tuning to a specific value.

Decision processes based on differencing followed by accumulation have also been reported in humans using indirect neuroimaging methods. Most notably, when humans are asked to decide whether a noisy image depicts a face or a house, left dorsolateral prefrontal cortex activity is proportional to the difference between activation in the fusiform face area and in the parahippocampal place area and predicts behavioral performance (Heekeren et al., 2004). Thus, this region, inscribed in a network of areas involving parietal and prefrontal regions, appears engaged in decision by evidence accumulation in humans. It seems likely that the same decision network would be involved in numerical tasks, but this has not been firmly demonstrated yet.

\section{Symbolic and nonsymbolic numbers}

Up to now, I have considered only the processing of nonsymbolic numerosities. I now turn to the processing of symbolic stimuli such as numbers presented as Arabic numerals or as written or spoken numerals.

\section{Theory}

How do written and spoken symbols come to have meaning? The symbol grounding problem consists in understanding how arbitrary shapes can ever acquire genuine meanings, over and above a mere network of relations to other symbols (Harnad, 1990). In the special case of numbers, I have proposed a simple solution to this grounding problem (Dehaene, 1997; see also Gelman and Gallistel, 1978). The nonsymbolic representation of 
numerosity is universally present in infants and adults of all cultures, and precedes the acquisition of linguistic symbols for numbers. When we learn number symbols, we simply learn to attach their arbitrary shapes to the relevant nonsymbolic quantity representations. Thus, the symbol ' 3 ' comes to evoke the very same representation that would be evoked by a set of three dots, namely a Gaussian distribution of activation over numerosity detector neurons. In neurophysiological terms, in the course of learning the meaning of numerals, neuronal assemblies involved in auditory and visual symbol analysis (respectively left superior temporal and bilateral ventral occipito-temporal cortices) must develop connections, direct or indirect, to neuronal assemblies in the depth of the intraparietal cortex.

According to an extreme version of this hypothesis, symbolic and nonsymbolic arithmetic tasks should then be captured by a single mathematical model with identical parameters including the internal weber fraction $w$. This hypothesis might be appropriate for certain experiments in which monkeys and apes are trained to recognize arabic digits and to attach them to the relevant numerosities by a pure process of association (Matsuzawa, 1985; Washburn and Rumbaugh, 1991; Tomonaga and Matsuzawa, 2002). Indeed, in those experiments, error rates are consistent with the use of estimation strategies comparable to those captured by the above mathematical model. However, there are reasons to believe that the situation might be more complex in humans. The acquisition of numerical symbols seems to provide access to a new level of competence for exact arithmetic. This conclusion is supported by several pieces of evidence. Cross-culturally, subjects whose culture has very few number words fail in exact arithmetic tasks that children in our culture easily perform (Gordon, 2004; Pica et al., 2004). Developmentally, the acquisition of counting is accompanied by a sudden emergence of precise responding, for instance in the 'give-a-number' task (Wynn, 1992b). Finally, neuroimaging and neuropsychological data indicate an association of exact arithmetic tasks with linguistic codes (Dehaene et al., 1999; Lemer et al., 2003).

To account for these unique consequences of acquiring symbolic information, a minimal assumption might be that the precision of the quantity code is modified by the acquisition of number symbols (Dehaene, 1997). Through interaction with a precise system, where each number $n$ is distinguished categorically from its neighbours $n-1$ and $n+1$, the tuning curves of numerosity detector neurons would become narrower, and the number line representation would crystallize into categorically distinct domains (Pica et al., 2004). In keeping with Steven Kosslyn's hypothesis of hemispheric specialization for categorical versus coordinate relations (Kosslyn et al., 1989), one might expect this refinement of numerical precision to occur mostly in the left hemisphere, which is also in more direct connection to linguistic symbols. For mathematical modeling purposes, we would then assume that all of the above formulas, developed for nonsymbolic numerosities, continue to hold for symbolic number processing, merely with a smaller value of the Weber fraction $w$.

While this is a viable model, a recent neural network simulation suggests that exposure to symbols may induce even more changes to the numerosity network (Verguts and Fias, 2004) (see Figure 24.5). Verguts and Fias used nonsupervised learning in a network 
exposed either to numerosity information alone, or to numerosity paired with an approximate symbol (coarsely approximating a child's inputs during acquisition of number words). Each symbolic input was coded by an arbitrary discrete unit. When nonsymbolic information alone was presented, the network developed numerosity detectors similar to Nieder and Miller's neurons and possessing all of the key properties of the standard model (skewed tuning curves on a linear axis, which become Gaussian when plotted on a logarithmic axis). Crucially, after pairing with symbolic information, the same numerosity detector units became tuned to symbols as well. Yet, there were two key differences between the unit's responses to symbolic and nonsymbolic inputs (see Figure 24.5). First, the tuning curves for symbolic inputs were much sharper. The simulated neurons essentially have a discrete, all-or-none peak of firing for their preferred value (thus each neuron cares mostly about a single, precise number), but they also show a shallow surrounding area of local preference for neighbouring numbers. Second, the tuning curves no longer broaden as the numbers increase (Weber's law), rather they have a fixed width for all numbers tested (1-5). Thus, the network develops a new type of representation, linear with fixed variability.

What I find most interesting in Verguts and Fias's proposal is that the very same neurons are involved in coding the quantity meaning of symbolic and nonsymbolic numerical information. The predictions for neural recordings are clear. When tested with symbolic and nonsymbolic inputs, each neuron would show the same preferred quantity in both domains. Only the tuning curve would be narrower for symbolic than for nonsymbolic
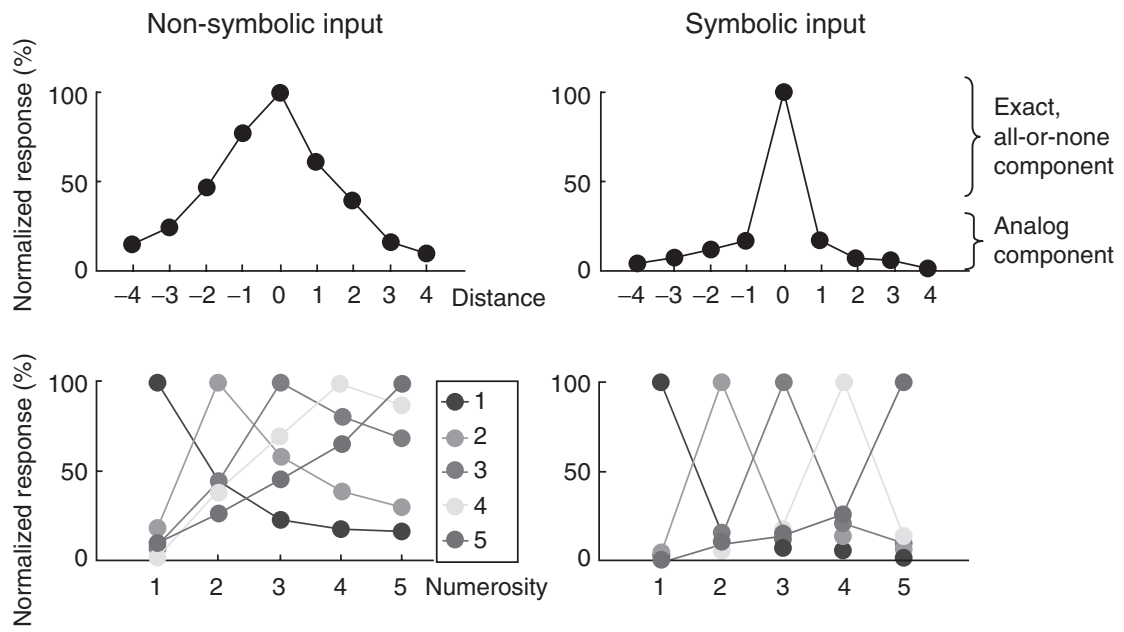

Figure 24.5 Numerical coding scheme emerging from Verguts and Fias's (2004) neuronal network after exposure to paired symbolic and nonsymbolic numerosities. When presented with nonsymbolic numerosities (left), the simulated neurons show broad distance-dependent tuning curves (top) that increase in width when plotted on a linear numerosity scale (bottom). When presented with symbolic numerals (right), the same neurons show sharp tuning curves (top) with fixed width as the number increases (bottom). 
inputs, and this difference would increase as the numbers become larger. The fact that the same neurons are involved means that there should be partial transfer of learning: if we learned to perform exact arithmetic tasks, using quantities derived from symbolic inputs, the same circuits would then be capable of performing the same operations approximately when nonsymbolic inputs are presented. Verguts and Fias's model thus proposes a concrete implementation of how a single brain area can be involved in both approximate and exact calculation modes (Dehaene, 1992; Dehaene and Cohen, 1995).

Verguts and Fias's proposal can be further extended. It seems possible that only some of the numerosity detector neurons acquire this more precise 'numerical receptive field' as a result of being associated with symbols. Even in educated human adults, some numerosity detector neurons, particularly in the right hemisphere, might keep their approximate tuning curves. These neurons might then encode the approximate meanings of 'round numbers' such as ten, fifteen, or a dozen (Dehaene and Mehler, 1992). The existence of an intermediate range of such neurons, with variable tuning precision, might explain the linguistic universal of two-numeral constructions (e.g. 'ten twelve books', 'ten fifteen books', 'ten twenty books'), by which we can refer symbolically not only to a quantity, but also to its variable degree of precision (Pollmann and Jansen, 1996).

Do these possibilities exhaust the transformations induced to our semantic system by the acquisition of symbolic numerals? No. There is evidence that humans possess a rich semantic lexicon for numbers. This lexicon specifies at least the parity and divisibility properties of numbers (e.g. primes, powers of two, multiplication facts) (Dehaene et al., 1993), but also more anecdotal semantic information such as famous dates, brands, etc. (e.g. 1492, 747) (Cohen et al., 1994). At the moment, however, incorporating such complex semantic information is way beyond the scope of our simple mathematical model.

\section{Experimental evidence}

\section{Distance effects with symbolic numerals}

The hypothesis that symbolic numerals inherit many of the properties of the nonsymbolic numerosity representation has been validated in a large number of experiments. A first indication of its plausibility came from Moyer and Landauer's (1967) finding of a distance effect when subjects compared two arabic digits. Although there was no similarity between stimuli at the symbol level, the subjects' behavior indicated the use of a mental representation of quantity where conceptual similarity varies with quantity proximity. This observation was quickly extended to many tasks. Distance effects have been observed in two-digit numeral comparison (Hinrichs et al., 1981; Dehaene, 1989; Dehaene et al., 1990), with detailed characteristics that seemed largely compatible with the random-walk model (Link, 1990; Page et al., 2004; Sigman and Dehaene, 2005) (see below). Even when deciding whether two number symbols are the same or not, response times vary with numerical distance, suggesting a high automaticity of the symbol-to-quantity conversion pathway (Duncan and McFarland, 1980; Dehaene and Akhavein, 1995). 


\section{Stages of processing of symbolic numerals}

The notion that symbolic numerals, regardless of their notation, pass through successive stages of symbol recognition followed by conversion to a nonverbal quantity code in parietal cortex was verified in several publications. I initially tested this issue using eventrelated potentials (ERPs) and the additive-factors method (Dehaene, 1996). During a comparison task with numbers presented as written words or as arabic numerals, response times showed additive effects of three factors: notation used (arabic or verbal), numerical distance, and motor effector (left or right hand). Additivity is consistent with serial stages of (1) numeral recognition, (2), notation-independent quantity comparison, and (3) response programming and execution. ERPs revealed signatures of all three stages, with a fast spread of activation first in left and right ventral occipito-temporal regions involved in numeral recognition, then in bilateral parietal cortices, where a distance effect was found, and finally in motor cortex. The separation between notation-dependent but distance-independent numeral processing in ventral occipito-temporal cortex, and notation-independent but distance-dependent quantity processing in intraparietal cortex, was later replicated in fMRI (Pinel et al., 2001, 2004).

Recently, Mariano Sigman and I used another chronometric technique to examine the decomposition of the number comparison task into processing stages (Sigman and Dehaene, 2005, 2006). In a psychological refractory period (PRP) design, we asked subjects to perform the number comparison task together with another unrelated pitch categorization task with tones presented at variable SOAs relative to number onset. Using the locus of slack logic (Pashler, 1984), we showed that notation- and response-dependent stages of the task could be performed in parallel with stages of the tone task, but that the distance-dependent stage could not-it was performed strictly serially with the response decision stage of the tone task. We also showed that the response time distribution expected from the random-walk model (Equation 24.24) fitted the data superbly, and that this distribution was shifted accordingly when the numerical decision was delayed by the tone decision. This study thus confirms the parsing of the number comparison task into notation-dependent and quantity-dependent stages and suggests that only the latter involves a 'central system' or 'global workspace' that can only perform one operation at a time (Dehaene and Changeux, 2004).

\section{Cerebral convergence of symbolic and nonsymbolic information}

Manuela Piazza, Philippe Pinel and I recently used fMRI adaptation to test directly the hypothesis of common neural populations for symbolic numerals and nonsymbolic numerosities (Piazza et al., 2006). During several minutes, subjects attended to the repeated presentation, every $1200 \mathrm{~ms}$, of an approximate quantity presented either as a set of dots (e.g. 17, 18, or 19 dots) or as an arabic numeral (the numerals 17, 18 or 19). We verified that the BOLD fMRI signal adapted over the course of about $40 \mathrm{~s}$ in left and right intraparietal cortices, within regions isolated using an independent localizer scan (subtraction task). We then introduced sparse deviants which could be close or far quantities (e.g. 20 or 50), and could appear in the same or different notation. The results replicated our earlier finding that the intraparietal cortex signal shows a distance-dependent 
recovery from adaptation (Piazza et al., 2004). Crucially, they also showed that this recovery holds even when the notation changes. This finding suggests, indirectly, that there must be populations of numerosity detector neurons jointly activated by symbolic and nonsymbolic notations, so that they can be habituated by one and transfer this habituation to the other.

Interestingly, in left parietal cortex, the effect was asymmetrical. When adaptation was to dots and the deviants were arabic numerals, there was recovery of adaptation to far but not to close quantities. However, when adaptation was to arabic numerals and the deviants were dots, there was recovery of adaptation to both close and far quantities (e.g. adaptation to 17-18-19, recovery to both 20 and 50). This finding suggests, in keeping with Verguts and Fias's (2004) model, that the quantities evoked by Arabic numerals may be more precise than those evoked by nonsymbolic sets of dots, at least in the left hemisphere, and hence the neuronal populations adapted by Arabic stimuli were narrower than those evoked by dot presentations. In the future, fMRI adaptation could be used to test more directly Verguts and Fias's (2004) model by plotting the profile of the adaptation curve as a function of numerical distance, and using this profile to infer the neuronal tuning curves for symbolic and nonsymbolic stimuli (Piazza et al., 2004).

\section{Acquisition of symbolic numerals}

While several neuroimaging studies have observed nonsymbolic quantity representations in parietal cortex in young children (Temple and Posner, 1998; Cantlon et al., 2006), very few studies have examined the acquisition of number symbols. The theory that I have outlined stipulates that, in the course of development, an increasingly automatized connection develops between ventral regions for symbol shape identification and intraparietal regions for quantity representation. Consistent with this idea, a recent fMRI study (Rivera et al., 2005) has examined the evolution of brain activity with age (8-19 years) during a simple symbolic arithmetic task which was performed with equal accuracy at all ages. While most regions showed a decrease in brain activity, particularly in prefrontal cortex, suggesting a progressive automatization, only two regions showed an increase: left occipito-temporal cortex and left parietal cortex, exactly as predicted.

\section{Log-to-linear shift during development}

An interesting prediction unique to Verguts and Fias's model is that the acquisition of number symbols is accompanied by a change in the internal semantic representation. While the representation of nonsymbolic numerosity is logarithmically compressed, Verguts and Fias's model implies that the symbolic representation is linear. In their simulations, when activated by symbols, numerosity detector neurons have fixed tuning curves on a linear scale, suggesting that they encode a linear, fixed variability scale no longer subject to Weber's law. This prediction can be tentatively related to observations of a shift from a logarithmic to a linear mapping of number onto a spatial scale in the course of development (Siegler and Opfer, 2003). Siegler and Opfer asked their subjects (8-, 10or 12-year-old children, plus an adult group) to point to the locations of numbers on a spatially extended segment labelled from 1 to 100 (or from 1 to 1000). Children of all 
ages performed this task well; both monotonically mapped number onto space, in agreement with the hypothesis that numerical quantities are represented internally on an internal continuum analogous to a mental 'number line' (Hubbard et al., 2005). Furthermore, the older children (12-year-olds and adults) organized the numbers linearly, spreading them evenly on the spatial scale. However, the youngest children (8-10-year-olds) spontaneously implemented a compressive mapping which was well captured by a logarithmic function. For instance, they place 10 close to the middle of 1 and 100, and grouped all of the larger numbers towards the large end.

Siegler and Opfer's data cannot resolve whether this log-to-linear change occurs spontaneously in the course of development, or depends on exposure to language and education. However, we have recently obtained a similar logarithmic effect in adult Mundurukus, an Amazonian people with reduced number lexicon and access to education and tools (S. Dehaene, V. Izard, P. Pica and E. Spelke, unpublished data, see Pica et al., 2004). On a segment marked with a single dot at one end and a set of ten dots at the other, we asked the Mundurukus to map the quantities 1 through 10 presented as sets of dots, series of tones, Munduruku numerals, or Portuguese numerals. In all of these modalities, the Munduruku performed logarithmically, similarly to Siegler and Opfer's younger children. Thus, although what triggers the conceptual shift from logarithmic to linear in children remains unknown, the fact that it is not observed in adult Mundurukus, who have very few spoken and no written numerals, suggests that mere maturation is not sufficient. Some experience with symbolic inputs seems necessary, but other factors such as explicit mathematical education and experience with measurement cannot be excluded.

\section{Refined analyses of the symbolic comparison task}

As noted above, the random-walk model, once fitted to RT and error data, provides a fine-grained estimation of the nature of representation and decision processes. To directly evaluate how these processes are organized for symbolic numerals, and to compare with the above nonsymbolic comparison task, I have reanalyzed the data for my studies of comparison of two-digit arabic numerals (Dehaene et al., 1990). In this task, human subjects had to classify arabic numerals as larger or smaller than a fixed reference. Three experiments were performed: comparison with 55, with 65 or with 66 .

Figure 24.6 shows the analysis of the data for comparison with 65, using analysis strategy 1. Both the RTs and error rates showed a distance effect which conformed well to the theory (errors showing a sharper decrease with distance than RTs). Furthermore, the expected relation between RT and transformed errors was always highly significant $(p<<0.001)$, indicating that the random-walk model applies well to symbolic comparison. Finally, there was a highly significant increase in mean step size $\mu$ with numerical distance ( $p<<0.001$ ). With respect to the above nonsymbolic comparison data, however, two distinctive features were observed. First, the variations of $\mu$ across the target numbers, were slightly better predicted by the linear model (i.e. by the difference between the target number and the standard 65) than by the logarithmic model (i.e. by the log ratio of the standard and target). Second, the $\mu$ were no longer strictly proportional to the numerical difference, as they were for nonsymbolic stimuli (Figure 24.4). There was a 
Human subjects, comparison of Arabic numerals with standard 65

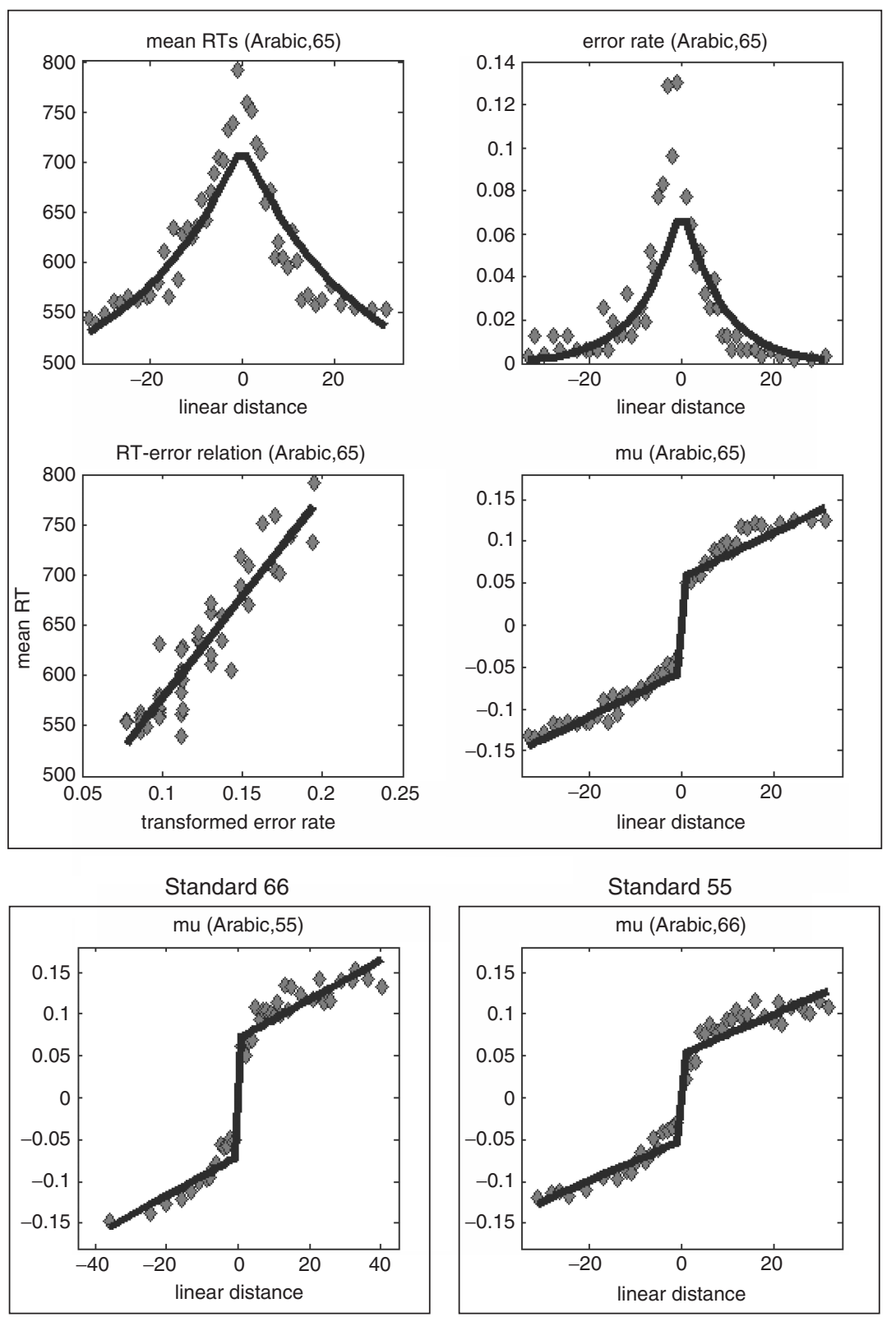

Figure 24.6 Symbolic comparison analyzed using the random-walk model (data reanalyzed from Dehaene et al., 1990). Human subjects compared two-digit arabic numerals to a fixed standard of 65 (top), 66 (bottom left), or 55 (bottom right). The format is the same as Figure 24.4, with the exception of a linear scale for target numbers (abscissa). Relative to numerosity comparison (Figure 24.4), arabic numeral comparison is much more precise, with error rates peaking at only $13 \%$ (top right panel). Examination of the $\mu$ parameter indicates that the information accumulated per unit of time increases with the difference between numbers (rather than their log ratio), and shows a discontinuity at the origin (information is accumulated at a fast rate even when the numerical difference is minimal). RT, reaction time. 
highly significant discontinuous offset as well ( $p<0.001$; compare Figures 24.4 and 24.6), indicating that as soon as the symbolic target was larger than the reference, even by one unit, a constant vote could be cast in favor of the 'larger' response.

These observations were replicated in analyses of two other symbolic comparison data sets (comparison with 55 and with 66; Dehaene et al., 1990; see Figure 24.6). Furthermore, in order to directly compare nonsymbolic and symbolic number processing within the same participants, Anna Wilson and I designed a new experiment in which, in different blocks, subjects had to compare numbers presented either as sets of dots or as two-digit arabic numerals (Dehaene and Wilson, unpublished data). In order to study Weber's law, on some blocks the numbers ranged from 11 to 39 and had to be compared to the reference 25, and in other blocks they ranged from 41 to 69 and had to be compared to the reference 55. In all cases, a highly regular distance effect was found, and the random-walk model provided an excellent fit of the data, including a highly significant RT-error relation. However, the recovered $\mu$ values showed that somewhat different numerical representations served as the basis for decision on symbolic and nonsymbolic trials (Figure 24.5). Two major differences were found. First, as above, on nonsymbolic trials the $\mu$ varied continuously with the numerical distance between the target and the standard (the range of targets was too small to tell whether this distance was better measured on a linear or log scale), but on symbolic trials there was again a clear discontinuous component. Second, when the magnitude of the numbers more than doubled (from standard 25 to standard 55), performance in the nonsymbolic block dropped, and the mean randomwalk step size $\mu$ decreased by a factor of about two (Weber's law) — but no such decrease was observed for symbolic numerals, where it was just as easy to compare numbers around 25 as around 55 .

What are the implications of these findings? The profile of mean step size $\mu$ suggests that two sources of information contribute to the decision-making process during symbolic number comparison. The first of these components grows, apparently linearly, with the difference between the target and the standard-this is a classical component of quantity-based evidence, similar to that found with nonsymbolic stimuli, except that the underlying continuum seems to be linear rather than logarithmic. The second component is all-or-none and provides a discrete vote for the larger response whenever the number is larger than the standard, or for the smaller response otherwise.

At first, the existence of this second component seems somewhat paradoxical. If such an accurate signal is available, why cannot the subject respond immediately, without being affected by the proximity of quantities? According to the random-walk model, the problem for the decision system is to extract the decision-relevant signals from other sources of noise. Assuming that numerosity detector neurons that have become very precise with symbolic exposure are intermixed with others that have remained imprecise, and that all are pooled together into the decision process, one would obtain precisely the observed summation of an exact, all-or-none signal and a distance-dependent analog signal. In Verguts and Fias's (2004) model, such exact and analog components are in fact present in the tuning curve of individual neurons (Figure 24.5). It would thus seem that this model can provide an excellent account of the data in Figures 24.6 and 24.7. 
Non-symbolic comparison
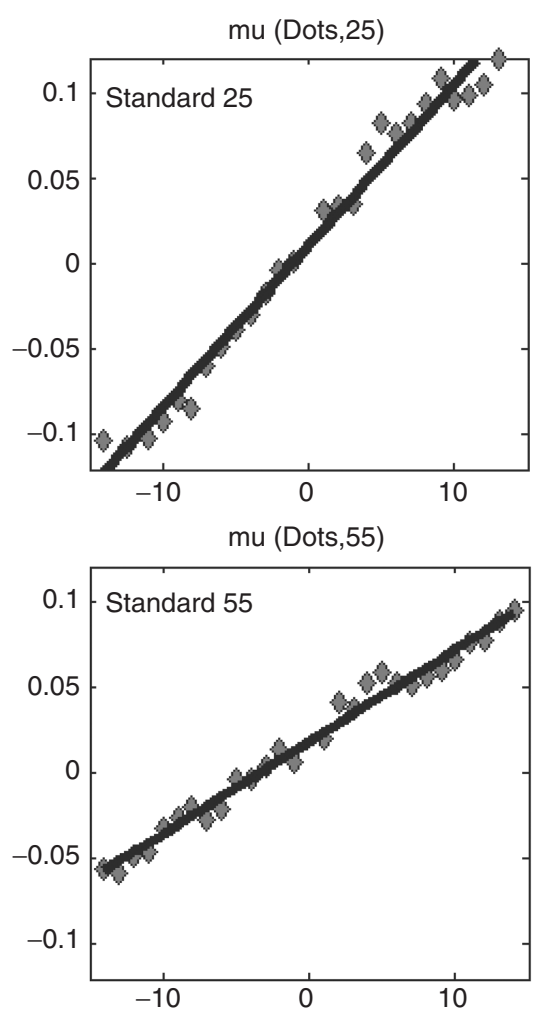

Symbolic comparison

mu (Arabic,25)

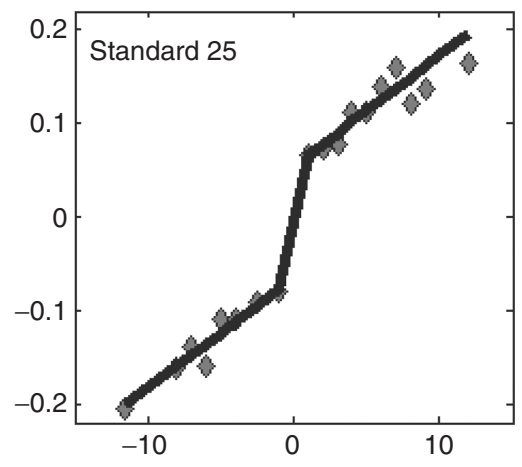

mu (Arabic,55)

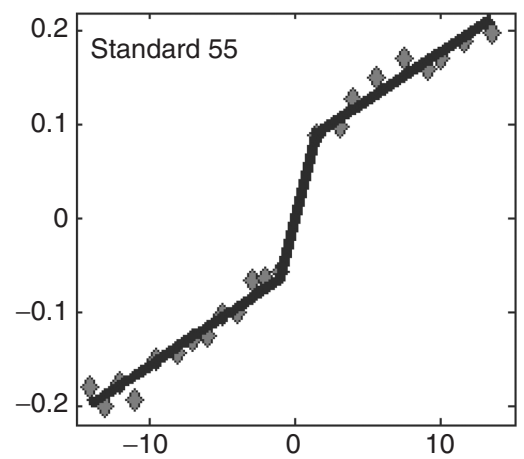

Figure 24.7 Replication of the differences between symbolic and nonsymbolic comparison within the same subjects (A. Wilson and S. Dehaene, unpublished data). The panels show the recovered $\mu$ for each of four distinct experimental blocks: comparison with standard 25 or 55 , and with targets presented as sets of dots or as two-digit arabic numerals. Note that for simplicity, all data are plotted as a function of the linear distance between target and standard (although with nonsymbolic targets, a slightly better fit is obtained with log ratio). The key differences are (1) the amount of information per unit of time is subject to Weber's law for nonsymbolic targets, but not for nonsymbolic targets; (2) a discontinuity is present at the origin for symbolic, but not for nonsymbolic targets.

I also used strategy 2 to fit the EZ-diffusion model to the symbolic number comparison data. The findings for the mean random-walk step size $\mu$ were unchanged, but the EZ-diffusion also allowed for an estimation of the parameters $T_{0}$ (nondecision time) and $\theta$ (decision threshold) separately for each target. I did not observe any significant variation of the decision threshold $\theta$, in keeping with the idea that this parameter is fixed prior to target presentation (however, see Botvinick et al. (2001) and Bogacz et al. (2006) for suggestions as to how this parameter may change from trial to trial). However, the nondecision time $T_{0}$ was systematically $70-100 \mathrm{~ms}$ slower for target numerals that fell within the decade of the standard (e.g. standard 65, targets between 60 and 69) compared 
to target numerals outside this decade. This aspect of the results resolves an older controversy concerning the origins of discontinuities in number comparison. The hypothesis of a digital-to-analog conversion during symbolic comparison suggested that RTs should be a smooth, continuous function of numerical distance. However, the results actually revealed small RT discontinuities at the boundaries of the decade of the standard (Hinrichs et al., 1981; Dehaene et al., 1990). The random-walk decomposition now shows clearly that these discontinuities arise outside of the decision system. In my analyses, the $\mu$ values that serve as inputs to the decision process never showed any discontinuity at decade boundaries, compatible with the hypothesis that decision is based on an analog quantity representation. However, the nondecision time showed a discontinuity, presumably imputable to a perceptual delay when the target number starts with the same decade digit as the standard and the subjects presumably have to orient more attention to the units digit.

In summary, the symbolic code for numbers appears to have both a perceptual cost (the need for longer perceptual analysis of the digital content of the stimulus) and a decision advantage (the decision is more precise and appears to be based, at least in part, on exact, all-or-none numerical information rather than solely on analog quantity information). All of the observed differences between symbolic and nonsymbolic number processing are compatible with Verguts and Fias's (2005) model of a narrowing and linearization of the tuning curves of numerosity detectors with exposure to symbolic inputs. ${ }^{7}$

\section{Simple calculations}

A final issue concerns how the number representation is used in simple arithmetic calculations such as addition or subtraction. Let it be clear from the outset that this part of the theory is much less developed. Here I focus exclusively on calculation with nonsymbolic numerosities, where some predictions can be made concerning error rates. Proposals as to how the theory might be extended to symbolic arithmetic will be briefly considered at the end.

A challenge to any theory of arithmetic is that a variety of tasks has been used to probe nonsymbolic calculation. Typically, subjects are shown two successive numerosities, either merely juxtaposed (Cordes et al., 2003; Lemer et al., 2003) or integrated into an animation that suggests an arithmetic operation of addition or subtraction (e.g. two sets of dots being added into a box) (Wynn, 1992a; McCrink and Wynn, 2004; Pica et al., 2004; Barth et al., 2005, 2006; McCrink et al., 2006). Subjects mentally compute the corresponding result and respond using one of several modes. They may be presented with a third number, and asked to compare it explicitly with their result, either using

7 Verguts and Fias further suggest that the symbolic number priming effect (Dehaene et al., 1998; Koechlin et al., 1999; Naccache and Dehaene, 2001) supports the model inasmuch as the size of the RT priming effect seems to vary with the linear distance between numbers rather than with their ratio (Reynvoet et al., 2002). 
mean and SD of RT (Arabic,65)
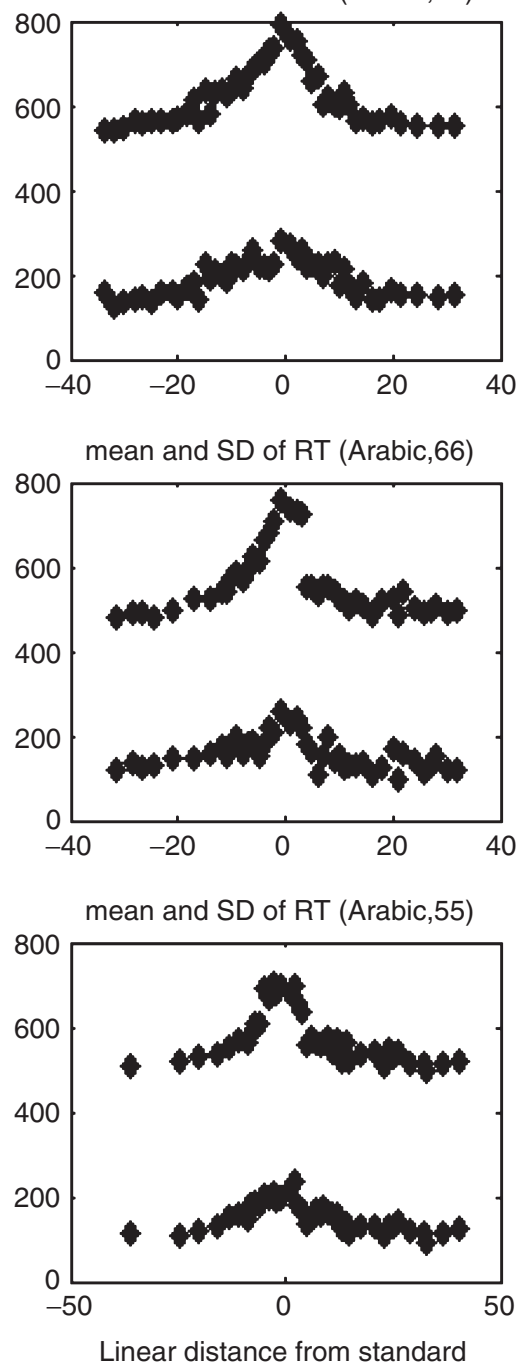

To (Arabic,65)

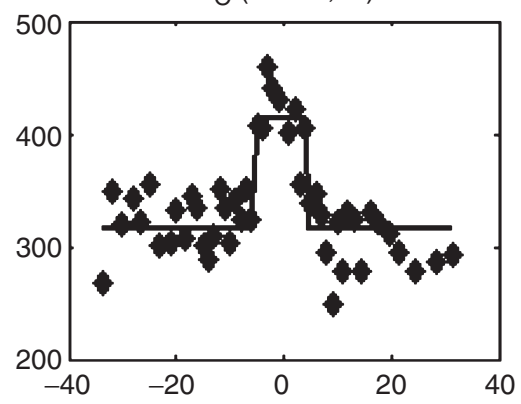

To (Arabic,66)
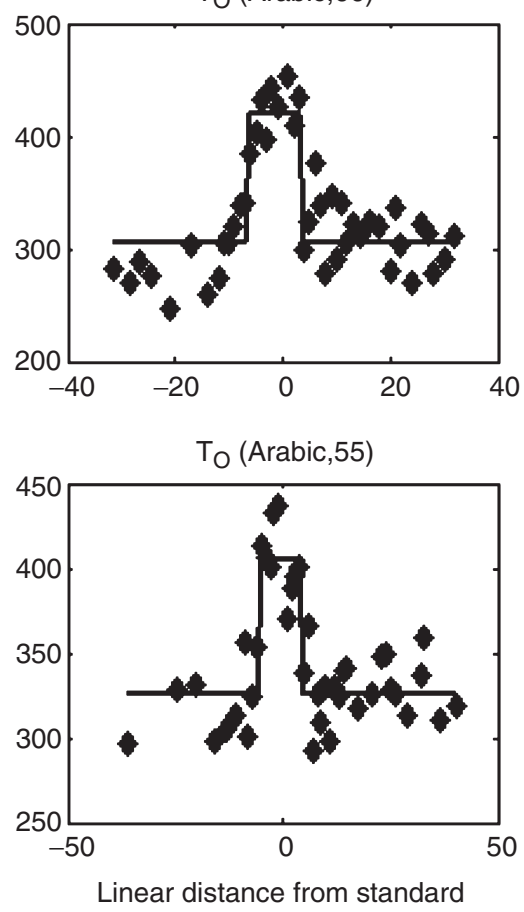

Figure 24.8 Mean and standard deviation (SD) of reaction time (RT) (left) and estimated nondecision time $T_{0}$ (right) in three experiments of two-digit number comparison analyzed with Wagenmakers' (2006) EZ-diffusion strategy for random-walk model identification. In all experiments, $T_{0}$ shows a clear discontinuity when the target crosses the decades boundary of the standard for comparison, suggesting an added perceptual cost of having to closely analyze the units digit. That this effect can be entirely attributed to nondecision processes is clearly visible in the graphs on the left, when the mean RT shows a sudden increase without any concomitant change in the standard deviation. 
larger-smaller comparison (Pica et al., 2004; Barth et al., 2005, 2006) or same-different judgment (McCrink et al., 2006). In preverbal infants, an implicit surprise reaction can be recorded when the outcome does not match the expected value (Wynn, 1992a; McCrink and Wynn, 2004). Educated subjects can also be asked to name the correct value or to point to it (Pica et al., 2004).

\section{Theory}

My first postulate will be that the decision components of all of these tasks are performed according to above-described mechanisms of larger-smaller, same-different, and numerosity labelling judgments (see in particular Equations 24.8-12 and 24.15). The sole difference is that the basis for the subjects' response is no longer an externally given quantity $n$, but rather an internally computed quantity, the result of an internal transformation on external inputs $n_{1}$ and $n_{2}$ which approximates the requested operation $n_{1}+n_{2}$ or $n_{1}-n_{2} \cdot{ }^{8}$ Assuming that the outcome $n$ is also represented internally by a Gaussian random variable $X$, the only remaining issue is how the mean and standard deviation of this variable vary as a function of the mean and standard deviation of the operands $n_{1}$ and $n_{2}$, separately for addition and for subtraction.

In the absence of any knowledge of the mechanisms of the internal transformation and their possible biases, the simplest normative model is one in which the subjects' mental estimations are unbiased, and thus the mental representation $q(n)$ of the result $n$ of an addition or subtraction operation is, on average, placed at the appropriate location on the number line:

$$
q_{+}(n)=q\left(n_{1}+n_{2}\right) \text { and } q_{-}(n)=q\left(n_{1}-n_{2}\right)
$$

where $q$ is the $\log$ function. Note that the implementation of the internal transformations implied by Equation 24.27 is not trivial. It is not the logarithms of the operands themselves that must be added or subtracted, otherwise the result would be multiplication or division. Rather, the logarithmic compression of the number line must somehow be 'undone' before the addition or subtraction operation is computed. In spite of its apparent complexity, such a computation is clearly within reach of simple neural networks that learn to extract simple 'basis functions' and can function as arbitrary interpolators (Denève et al., 2001). In fact, a computation formally similar to addition and subtraction is implemented by the parietal spatial updating mechanism (Duhamel et al., 1992), which uses eye movement direction and amplitude to remap memories of saccade targets. The computation performed is analogous to vector addition, and is not impeded by the fact that the retinotopic map shows logarithmic foveal expansion. It is a highly intriguing anatomical fact that the areas involved in this vector addition process, VIP and LIP, overlap

8 In its most general version, this hypothesis states that any number obtained as the output of some arithmetic calculation or decision can be re-used as the input into another. How such 'piping' of intermediate results occurs in the human brain remains highly unclear. My speculation is that it requires a central exchange system or global workspace, presumably involving prefrontal cortex, whose operation is necessarily serial and consciously controlled (see Dehaene and Changeux, 2004). 
with those involved in number representation (Dehaene et al., 2003; Nieder and Miller, 2004). This anatomical relation tentatively suggests a possible 'recycling' of the spatial remapping VIP-LIP circuitry for computationally similar arithmetic transformations (Dehaene, 2005; Hubbard et al., 2005).

Can one make any predictions concerning the precision of such an addition or subtraction result? Clearly, the precision of the outcome should depend on the initial precision of the operands. Both the log-Gaussian model and the scalar variability model predict that number encoding is subject to Weber's law, i.e. variability proportional to the mean. If the random variables encoding the two numbers are stochastically independent, then their variances should add, leading to:

$$
\operatorname{var}_{\varepsilon}(n)=\operatorname{var}\left(n_{1}\right)+\operatorname{var}\left(n_{2}\right)
$$

where, according to Weber's law, $\operatorname{var}\left(n_{1}\right)=\left(w n_{1}\right)^{2}$ and $\operatorname{var}\left(n_{2}\right)=\left(w n_{2}\right)^{2}$.

In this equation, $\varepsilon$ is +1 for addition, and -1 for subtraction. The equation makes it clear that the precision of these operations should depend solely on the size of the operands. For instance, the same final precision is predicted for the operations $24+8$ and $24-8$, although the outcomes are centered on 32 and 16 respectively. An alternative equivalent formulation of this property states that, for equal results $n$, addition should always be more precise than subtraction.

Two refinements of Equation 24.28 have been proposed independently by Cordes et al. (2003) and by my collaborators and I (Barth et al., 2006; McCrink et al., 2006). First, there might be a covariance term if $n_{1}$ and $n_{2}$ are not estimated independently. For instance, subjects might compare $n_{2}$ with $n_{1}$, or in some subtraction displays with moving objects they might notice that some of the objects composing set $n_{1}$ appear to leave the display during presentation of $n_{2}$ (McCrink et al., 2006). Such sources of covariance in the estimation of $n_{1}$ and $n_{2}$ would lead to a better precision on the outcome of $n_{1}-n_{2}$ than expected based on the estimation of $n_{1}$ and $n_{2}$ in isolation—and to a worse precision on the outcome of $n_{1}+n_{2}$.

A second source of variance might come from the operation itself and, in particular, the need to temporarily store the result $n$. In the absence of a more precise characterization of the arithmetic mechanism itself, both Cordes et al. (2003) and Barth et al. (2006) have proposed to subsume these effects under an additional term with Weberian variability on the outcome $n$.

With those two refinements, the variance of an operation result becomes:

$$
\operatorname{var}_{\varepsilon}(n)=\operatorname{var}\left(n_{1}\right)+\operatorname{var}\left(n_{2}\right)+2 \varepsilon \operatorname{cov}\left(n_{1}, n_{2}\right)+\lambda \operatorname{var}\left(n_{1}+\varepsilon n_{2}\right)
$$

where according to Weber's law, $\operatorname{var}\left(n_{1}\right)=\left(w n_{1}\right)^{2}, \operatorname{var}\left(n_{2}\right)=\left(w n_{2}\right)^{2}, \operatorname{var}\left(n_{1}+\varepsilon n_{2}\right)=$ $w^{2}\left(n_{1}+\varepsilon n_{2}\right)^{2}$, and $\lambda$ is a scaling factor which indicates how much additional imprecision is due to calculation, over and above the intrinsic imprecision due to the internal representation. Yet, a difficulty with this equation is that, at present, no one has proposed a precise form for the covariance term, thus limiting its practical applicability. 
Once precise hypotheses are made about the mean (24.27) and variance (24.28 or 24.29) of an arithmetic result, it is relatively easy to model specific tasks by modifying the above Equations 24.8-24.12, and 24.15. For instance, if the subject is asked to decide whether the arithmetic result $n_{1}+n_{2}$ is larger or smaller than a third numerosity $n$, under the hypotheses of Equation 24.29 and assuming stochastic independence (no covariance term), Equation 24.10 then becomes:

$$
\begin{aligned}
& P_{\text {correct }}\left(n_{1}, n_{2}, n_{3}\right)=\int_{0}^{+\infty} \frac{e^{-\frac{1}{2}\left(\frac{x-\left(n_{1}+\in n_{2}-n_{3}\right)}{w \sqrt{n_{1}^{2}+n_{2}^{2}+n_{3}^{2}+\lambda^{2}\left(n_{1}+\epsilon n_{2}\right)^{2}}}\right)^{2}}}{\sqrt{2 \pi} w \sqrt{n_{1}^{2}+n_{2}^{2}+n_{3}^{2}+\lambda^{2}\left(n_{1}+\in n_{2}\right)^{2}}} d x \\
&=\int_{0}^{+\infty} \frac{e^{-\frac{1}{2}\left(\frac{x-(r-1)}{w \sqrt{1+r^{2}+2 \alpha(\alpha-1)+\lambda^{2}}}\right)^{2}}}{\sqrt{2 \pi} w \sqrt{1+r^{2}+2 \alpha(\alpha-1)+\lambda^{2}}} d x \\
& \text { with } r=\frac{n_{3}}{n_{1}+\varepsilon n_{2}} \text { and } \alpha=\frac{n_{1}}{n_{1}+\varepsilon n_{2}}
\end{aligned}
$$

This equation was used by Barth et al. (2006) and, with the simplifying assumption $\lambda=0$, by Pica et al. (2004) to model their numerosity addition and subtraction tasks.

\section{Experimental evidence}

The ability to combine two numerosities into simple addition and subtraction operations has been demonstrated in preverbal human infants using a surprise paradigm in which infants look longer when an arithmetically impossible outcome is presented (Wynn, 1992a; Koechlin et al., 1997). This competence was initially demonstrated for very small numerosities $(1+1$ or $2-1)$ around $4 \frac{1}{2}$ months of age, and it has recently been extended to large numerosities $(5+5$ and 10-5) in 9-month-old infants (McCrink and Wynn, 2004).

A simple prediction, common to all models, is that performance in such tasks should depend primarily on the ratio of the arithmetic result to the proposed result. This ratio dependence has not been tested in infants yet, but it has been observed in 5-year-olds (Barth et al., 2005) and adults (Pica et al., 2004; Barth et al., 2006). Barth et al. (2005) showed that 5-year-olds could perform a cross-modal addition combining visually and auditorily presented numerosities, thus confirming that the computation occurs at an abstract numerosity representation level rather than between visual images of sets.

Recently, a few studies have begun to generate quantitative data appropriate for testing the more precise predictions of the model (Cordes et al., 2003; Pica et al., 2004; Barth et al., 2006; McCrink et al., 2006). Pica et al. (2004) showed that Equation 24.28 (without 
covariance or $\lambda$ terms) accurately fitted the data from Western and Amazonian subjects performing numerosity addition and subtraction tasks. Furthermore, a prediction of that equation, the fact that performance should be more precise for addition than for subtraction, was verified by Barth et al. (2006). However, Barth et al. (2006) also noted an important deviation which suggests rejection of this simple model. Somewhat counterintuitively, Equation 24.28 predicts that the precision on the result $n$ of an addition $n_{1}+n_{2}$ can be better than if $n$ had been presented directly to the subject. This is because, if the variances of $n_{1}$ and $n_{2}$ add, the standard deviation of the result can be proportionally smaller than the standard deviation of the initial values (by a factor which can be as large as 2). Barth et al. (2006), however, always observed worse performance in addition plus comparison (deciding whether $n_{1}+n_{2}$ is larger or smaller than $n_{3}$ ) than in comparison alone. Similar observations were made by Cordes et al. (2003). Both authors suggest the need for an additional $\lambda$ term reflecting the additional variance introduced by the arithmetic operation and the maintenance of the result $n$ in memory. The Barth et al. (2006) data were accurately fitted by letting $\lambda=1.3$.

Can one similarly prove the necessity of the covariance term? For subtractions involving large operands but a small result (e.g. $32-28$ ), the model without covariance predicts that final precision should be very poor, but this is not the case (Cordes et al., 2003; McCrink et al., 2006). McCrink et al. (2006) explicitly compared performance on matched pairs of operations such as $24+8$ and $24-8$. The model without covariance or $\lambda$ term predicts equal final precision, but this was clearly false: in all cases subtraction was more precise, compatible with the covariance model. Unfortunately, the absence of a simple comparison baseline, without addition or subtraction, did not make it possible to further specify the parameters of the model.

Koleen McCrink and I also made a new observation that questions more deeply the above theoretical framework (McCrink et al., 2006). In our experiments, over a large number of trials, we repeatedly presented subjects with the same operation (e.g. $24+8$ ), each time paired with a different outcome (e.g. 13, 16, 20, 24, 32, 40,48). Subjects had to decide whether these outcomes were correct or not. In this way, we were able to trace a curve that indicated which numerosity subjects judged as the most likely outcome of a given operation. Surprisingly, although this value always fell close to the correct arithmetic outcome it presented a systematic bias. Additions were slightly overestimated, and subtractions were notably underestimated (in retrospect, a similar trend is perceptible in Cordes et al., 2003). This observation tentatively suggests that the number line may be submitted to a 'representational momentum' effect similar to that found for physical motion (Freyd and Finke, 1984) or motion on the pitch continuum (Freyd et al., 1990): during an addition operation, while moving towards larger numbers, the internal representation would 'move too far' towards the large end of the number line- and a converse shift towards small numbers would occur during subtraction. While the numerical momentum effect thus supports the hypothesis that arithmetic is analogous to motion on the number line, and that similar circuity is recruited for both numerical and spatial updating (Hubbard et al., 2005), this finding also complicates the search for a simple mathematical model of elementary arithmetic. 


\section{Open issues}

A central question concerns the relation of these nonsymbolic arithmetic abilities to those deployed in calculation with arabic digits. The dominant view is that symbolic arithmetic is based on 'two calculation sytems' (Dehaene and Cohen, 1991), one based on quantity manipulations and used for nonsymbolic numerosities, and the other based on purely formal processes of symbol manipulation and explicit memory retrieval (Ashcraft, 1992). The hopes of capturing such a complex set of pathways by simple mathematical equations are dim. Nevertheless, it has been suggested that the simplest mathematical operations, particularly subtraction, may rely primarily on nonsymbolic quantity manipulations (Gallistel and Gelman, 1992; Cohen and Dehaene, 2000; Cohen et al., 2000). The observation of highly regular effects of number size (Ashcraft, 1992), of clear distance effects when subjects are asked to evaluate the correctness of a proposed operation outcome (Ashcraft and Stazyk, 1981), and of joint impairments of symbolic and nonsymbolic addition after a small left parietal hemorrhage in a patient with dyscalculia and Gerstmann's syndrome (Lemer et al., 2003) hint that elementary symbolic arithmetic relies heavily on quantity representations of the type described in this chapter. The emergence of new ideas concerning the encoding of symbolic and nonsymbolic quantities (Verguts and Fias, 2004) and of new possibilities to visualize the cerebral mechanisms of arithmetic, perhaps down to the single-neuron level (Nieder et al., 2002; Nieder and Miller, 2003, 2004), invites new reflections on this topic.

\section{Conclusion}

I have presented a simple but powerful mathematical theory of number representation and manipulation. As mentioned in the introduction, although putting it all together has required some ingenuity, none of its elements are particularly original. The theory has been developed over the years by many groups, and it draws heavily on pre-existing developments in signal detection theory and random-walk models of decision making. Yet the virtue of such a theorizing effort is three-fold. First, it presents a homogeneous and formal description of behavior in simple numerical tasks, complete with detailed equations that can be quantitatively compared with human and animal performance. Second, it proposes bridging laws linking those behavioral approaches to the underlying neuronal mechanisms. With the emergence of single-neuron studies of arithmetic, those bridging laws will become increasingly testable. Third, the theory is simple enough that it can serve as a minimal 'standard model' on which to base further improvements.

An interesting issue is to what extent the present theory could be generalized outside the numerical domain. The coding principles outlined in 'Numerosity representation' are clearly not unique to numbers - in fact they make use of Gaussian tuning curves, population coding, and psychophysical decision mechanisms that have been used for years in coding of perceptual dimensions such as movement direction and spatial coordinates. Furthermore, mechanisms for combining two such values into simple arithmetic-like operations exist in many animal species which are known to compute vector sums, temporal differences or reward rates (Gallistel, 1990). It has been proposed that the 
general circuitry linking parietal areas VIP and LIP is jointly used for vector sums and differences in the domains of space, time and number (Hubbard et al., 2005). Indeed, the coding and manipulation of spatial, temporal and numerical magnitudes might constitute an overarching function of the parietal lobes (Walsh, 2003). The present theory would readily extend to these domains. Still, one should remain aware that neuronal coding principles can also differ across domains, as exemplified by Gaussian tuning for number versus coding by monotonically varying firing rates for tactile frequency (Romo and Salinas, 2003). The range of solutions available to neuronal networks for solving arithmetic-like problems, as well as the reason for choosing one over the other, remain to be thoroughly theorized (see Verguts, 2006; Salinas 2006).

Even within the numerical domain, attempting to build an integrative theory has revealed at least four areas where our knowledge is insufficient: How do we encode symbolic numerals? What factors generate a switch from logarithmic to linear representations of quantity in the course of development? How do we compute simple arithmetic transformations? And what is the global architecture which permits flexible chaining of operations and feeding of the results of one operation into another ('piping')? I have no doubt that the present minimal propositions will serve as a useful target for experimenters, and hope that the advancement of numerical cognition will be such that they will have to be quickly replaced by more refined ideas.

\section{Acknowledgements}

This work was supported by INSERM, CEA, and a Centennial Fellowship from the James S. McDonnell Foundation. I gratefully acknowledge extensive discussions with many colleagues over the past twenty years, particularly J. Mehler, G. Dehaene-Lambertz, E. Dupoux, L. Cohen, J. P. Changeux, R. Shepard, R. Gallistel, R. Gelman, E. Koechlin, L. Naccache, P. Pinel, E. Spelke, H. Barth, A. Nieder, V. Izard, P. Pica, M. Piazza, M. Sigman, A. Wilson, and E. Hubbard. I am extremely grateful to Jessica Cantlon and Elizabeth Brannon for letting me analyze their raw data.

\section{References}

Ashcraft, M. H. (1992) Cognitive arithmetic: a review of data and theory. Cognition, 44, 75-106.

Ashcraft, M. H. and Stazyk, E. H. (1981) Mental addition: a test of three verification models. Memory and Cognition, 9, 185-196.

Barth, H., La Mont, K., Lipton, J. and Spelke, E. S. (2005) Abstract number and arithmetic in preschool children. Proceedings of the National Academy of Sciences of the USA, 102, 14116-14121.

Barth, H., La Mont, K., Lipton, J., Dehaene, S., Kanwisher, N. and Spelke, E. (2006) Non-symbolic arithmetic in adults and young children. Cognition, 98, 199-222.

Bogacz, R., Brown, E., Moehlis, J., Holmes, P. and Cohen, J. D. (2006) The physics of optimal decision making: a formal analysis of models of performance in two-alternative forced-choice tasks. Psychological Review, 113, 700-765.

Botvinick, M. M., Braver, T. S., Barch, D. M., Carter, C. S. and Cohen, J. D. (2001) Conflict monitoring and cognitive control. Psychological Review, 108, 624-652.

Brannon, E. M. and Terrace, H. S. (2000) Representation of the numerosities 1-9 by rhesus macaques (Macaca mulatta). Journal of Experimental Psychology: Animal Behavior Processes, 26, 31-49. 
Brannon, E. M., Wusthoff, C. J., Gallistel, C. R. and Gibbon, J. (2001) Numerical subtraction in the pigeon: evidence for a linear subjective number scale. Psychological Science, 12, 238-243.

Cantlon, J. F. and Brannon, E. M. (2006) Shared system for ordering small and large numbers in monkeys and humans. Psychological Science, 17, 401-406.

Cantlon, J. F., Brannon, E. M., Carter, E. J. and Pelphrey, K. A. (2006) Functional imaging of numerical processing in adults and 4-y-old children. PLoS Biology, 4, e125.

Cohen, L. and Dehaene, S. (2000) Calculating without reading: unsuspected residual abilities in pure alexia. Cognitive Neuropsychology, 17, 563-583.

Cohen, L., Dehaene, S. and Verstichel, P. ( 1994) Number words and number non-words: a case of deep dyslexia extending to arabic numerals. Brain, 117, 267-279.

Cohen, L., Dehaene, S., Chochon, F., Lehéricy, S. and Naccache, L. (2000) Language and calculation within the parietal lobe: a combined cognitive, anatomical and fMRI study. Neuropsychologia, 38, 1426-1440.

Cordes, S., Gelman, R., Gallistel, C. R. and Whalen, J. (2001) Variability signatures distinguish verbal from nonverbal counting for both large and small numbers. Psychonomic Bulletin and Review, 8, 698-707.

Cordes, S., Gallistel, C. R., Gelman, R. and Latham, P. E. (2003) Nonverbal arithmetic in humans, Vancouver, BC. Paper presented at the Annual meeting of OPAM (Object Perception Attention and Memory), Vancouver, BC, November 2003.

Dayan, P. and Abbott, L. F. (2001) Theoretical Neuroscience: Computational and Mathematical Modeling of Neural Systems. MIT Press, Cambridge, MA.

Dehaene, S. (1989) The psychophysics of numerical comparison: a re-examination of apparently incompatible data. Perception and Psychophysics, 45, 557-566.

Dehaene, S. (1992) Varieties of numerical abilities. Cognition, 44, 1-42.

Dehaene, S. (1996) The organization of brain activations in number comparison: event-related potentials and the additive-factors methods. Journal of Cognitive Neuroscience, 8, 47-68.

Dehaene, S. (1997) The Number Sense. Oxford University Press, New York.

Dehaene, S. (2001) Subtracting pigeons: logarithmic or linear? Psychological Science, 12, 244-246.

Dehaene, S. (2002) Single-neuron arithmetic. Science, 297(5587), 1652-1653.

Dehaene, S. (2003) The neural basis of the Weber-Fechner law: a logarithmic mental number line. Trends in Cognitive Science, 7, 145-147.

Dehaene, S. (2005) Evolution of human cortical circuits for reading and arithmetic: the 'neuronal recycling' hypothesis. In Dehaene, S., Duhamel, J. R., Hauser, M. and Rizzolatti, G. (eds), From Monkey Brain to Human Brain, pp. 133-157. MIT Press, Cambridge, MA.

Dehaene, S. and Akhavein, R. (1995) Attention, automaticity and levels of representation in number processing. Journal of Experimental Psychology: Learning, Memory and Cognition, 21, 314-326.

Dehaene, S. and Changeux, J. P. (1993) Development of elementary numerical abilities: a neuronal model. Journal of Cognitive Neuroscience, 5, 390-407.

Dehaene, S. and Changeux, J. P. (2004) Neural mechanisms for access to consciousness. In Gazzaniga, M. (ed.), The Cognitive Neurosciences, 3rd edn, Vol. 82, pp. 1145-1157. Norton, New York.

Dehaene, S. and Cohen, L. (1991) Two mental calculation systems: a case study of severe acalculia with preserved approximation. Neuropsychologia, 29, 1045-1074.

Dehaene, S. and Cohen, L. (1994) Dissociable mechanisms of subitizing and counting: neuropsychological evidence from simultanagnosic patients. Journal of Experimental Psychology: Human Perception Performance, 20, 958-975.

Dehaene, S. and Cohen, L. (1995) Towards an anatomical and functional model of number processing. Mathematical Cognition, 1, 83-120.

Dehaene, S. and Mehler, J. (1992) Cross-linguistic regularities in the frequency of number words. Cognition, 43, 1-29. 
Dehaene, S., Dupoux, E. and Mehler, J. (1990) Is numerical comparison digital: analogical and aymbolic effects in two-digit number comparison. Journal of Experimental Psychology: Human Perception and Performance, 16, 626-641.

Dehaene, S., Bossini, S. and Giraux, P. (1993) The mental representation of parity and numerical magnitude. Journal of Experimental Psychology: General, 122, 371-396.

Dehaene, S., Naccache, L., Le Clec'H, G., Koechlin, E., Mueller, M., Dehaene-Lambertz, G., van de Moortele, P. F. and Le Bihan, D. (1998) Imaging unconscious semantic priming. Nature, 395, 597-600.

Dehaene, S., Spelke, E., Pinel, P., Stanescu, R. and Tsivkin, S. (1999) Sources of mathematical thinking: behavioral and brain-imaging evidence. Science, 284(5416), 970-974.

Dehaene, S., Piazza, M., Pinel, P. and Cohen, L. (2003) Three parietal circuits for number processing. Cognitive Neuropsychology, 20, 487-506.

Dehaene, S., Izard, V. and Piazza, M. (2005) Control over non-numerical parameters in numerosity experiments. Unpublished manuscript (available on www.unicog.org).

Deneve, S., Latham, P. E. and Pouget, A. (2001) Efficient computation and cue integration with noisy population codes. Nature Neuroscience, 4, 826-831.

Duhamel, J. R., Colby, C. L. and Goldberg, M. E. (1992) The updating of the representation of visual space in parietal cortex by intended eye movements. Science, 255, 90-92.

Duncan, E. M. and McFarland, C. E. (1980) Isolating the effects of symbolic distance and semantic congruity in comparative judgments: an additive-factors analysis. Memory and Cognition, 8, 612-622.

Feigenson, L., Dehaene, S. and Spelke, E. (2004) Core systems of number. Trends in Cognitive Sciences, 8, 307-314.

Freyd, J. J. and Finke, R. (1984) Representational momentum. Journal of Experimental Psychology: Learning, Memory and Cognition, 10, 126-132.

Freyd, J. J., Kelly, M. H. and DeKay, M. L. (1990) Representational momentum in memory for pitch. Journal of Experimental Psychology: Learning, Memory and Cognition, 16, 1107-1117.

Gallistel, C. R. (1990) The Organization of Learning. MIT Press, Cambridge, MA.

Gallistel, C. R. and Gelman, R. (1992) Preverbal and verbal counting and computation. Cognition, $44,43-74$.

Gelman, R. and Gallistel, C. R. (1978) The Child's Understanding of Number. Harvard University Press, Cambridge, MA.

Gibbon, J. (1977) Scalar expectancy theory and Weber's law in animal timing. Psychological Review, 84, 279-325.

Gibbon, J. and Fairhurst, S. (1994) Ratio versus difference comparators in choice. Journal of the Experimental Analysis of Behavior, 62, 409-434.

Gold, J. I. and Shadlen, M. N. (2001) Neural computations that underlie decisions about sensory stimuli. Trends in Cognitive Sciences, 5, 10-16.

Gold, J. I. and Shadlen, M. N. (2002) Banburismus and the brain: decoding the relationship between sensory stimuli, decisions and reward. Neuron, 36, 299-308.

Gordon, P. (2004) Numerical cognition without words: evidence from Amazonia. Science, 306, 496-499.

Green, D. and Swets, J. A. (1966) Signal detection theory and psychophysics. Krieger, New York.

Harnad, S. (1990) The symbol grounding problem. Physica D, 42, 335-346.

Hauser, M. D. and Carey, S. (2003) Spontaneous representations of small numbers of objects by rhesus macaques: examinations of content and format. Cognitive Psychology, 47, 367-401.

Heekeren, H. R., Marrett, S., Bandettini, P. A. and Ungerleider, L. G. (2004) A general mechanism for perceptual decision-making in the human brain. Nature, 431(7010), 859-862.

Hinrichs, J. V., Yurko, D. S. and Hu, J. M. (1981) Two-digit number comparison: use of place information. Journal of Experimental Psychology: Human Perception and Performance, 7, 890-901. 
Hubbard, E. M., Piazza, M., Pinel, P. and Dehaene, S. (2005) Interactions between number and space in parietal cortex. Nature Reviews Neuroscience, 6, 435-448.

Izard, V. (2005) Interactions between verbal and non-verbal numerical representations: theoretical and empirical approaches. PhD thesis, University of Paris VI, Paris.

Kim, J. N. and Shadlen, M. N. (1999) Neural correlates of a decision in the dorsolateral prefrontal cortex of the macaque. Nature Neuroscience, 2, 176-185.

Koechlin, E., Dehaene, S. and Mehler, J. (1997) Numerical transformations in five month old human infants. Mathematical Cognition, 3, 89-104.

Koechlin, E., Naccache, L., Block, E. and Dehaene, S. (1999) Primed numbers: exploring the modularity of numerical representations with masked and unmasked semantic priming. Journal of Experimental Psychology: Human Perception and Performance, 25, 1882-1905.

Kosslyn, S. M., Koenig, O., Barrett, A., Cave, C. B., Tang, J. and Gabrieli, J. D. E. (1989) Evidence for two types of spatial representations: hemispheric specialization for categorical and coordinate relations. Journal of Experimental Psychology: Human Perception and Performance, 15, 723-735.

Krueger, L. E. (1982) Single judgments of numerosity. Perception and Psychophysics, 31, 175-182.

Laming, D. R. J. (1968) Information Theory of Choice-reaction Times. Academic Press, London.

Lemer, C., Dehaene, S., Spelke, E. and Cohen, L. (2003) Approximate quantities and exact number words: dissociable systems. Neuropsychologia, 41, 1942-1958.

Link, S. W. (1975) The relative judgment theory of two choice response time. Journal of Mathematical Psychology, 12, 114-135.

Link, S. W. (1990) Modelling imageless thought: the relative judgment theory of numerical comparisons. Journal of Mathematical Psychology, 34, 2-41.

Link, S. W. (1992) The Wave Theory of Difference and Similarity. Erlbaum, Hillsdale, NJ

Lipton, J. and Spelke, E. (2003) Origins of number sense: large number discrimination in human infants. Psychological Science, 14, 396-401.

Lo, C. C. and Wang, X. J. (2006). Cortico-basal ganglia circuit mechanism for a decision threshold in reaction time tasks. Nat Neurosci, 9(7), 956-963

Machens, C. K., Romo, R. and Brody, C. D. (2005) Flexible control of mutual inhibition: a neural model of two-interval discrimination. Science, 307(5712), 1121-1124.

MacMillan, N. A. and Creelman, C. D. (1991) Detection Theory: A User's Guide. Cambridge University Press, Cambridge.

Mandler, G. and Shebo, B. J. (1982) Subitizing: an analysis of its component processes. Journal of Experimental Psychology: General, 111, 1-21.

Matsuzawa, T. (1985) Use of numbers by a chimpanzee. Nature, 315(6014), 57-59.

Mazurek, M. E., Roitman, J. D., Ditterich, J. and Shadlen, M. N. (2003) A role for neural integrators in perceptual decision making. Cerebral Cortex, 13, 1257-1269.

McCrink, K. and Wynn, K. (2004) Large-number addition and subtraction by 9-month-old infants. Psychological Science, 15, 776-781.

McCrink, K., Dehaene, S. and Dehaene-Lambertz, G. (2006) Moving along the number line: Operational momentum in non-symbolic arithmetic. manuscript submitted for publication.

Mechner, F. (1958) Probability relations within response sequences under ratio reinforcement. Journal of the Experimental Analysis of Behavior, 1, 109-121.

Minturn, A. L. and Reese, T. W. (1951) The effect of differential reinforcement on the discrimination of visual number. Journal of Psychology, 31, 201-231.

Moyer, R. S. and Landauer, T. K. (1967) Time required for judgements of numerical inequality. Nature, 215, 1519-1520.

Naccache, L. and Dehaene, S. (2001) The priming method: imaging unconscious repetition priming reveals an abstract representation of number in the parietal lobes. Cerebral Cortex, 11, 966-974. 
Nieder, A. and Miller, E. K. (2003) Coding of cognitive magnitude. Compressed scaling of numerical information in the primate prefrontal cortex. Neuron, 37, 149-157.

Nieder, A. and Miller, E. K. (2004) A parieto-frontal network for visual numerical information in the monkey. Proceedings of the National Academy of Sciences of the USA, 101, 7457-7462.

Nieder, A., Freedman, D. J. and Miller, E. K. (2002) Representation of the quantity of visual items in the primate prefrontal cortex. Science, 297(5587), 1708-1711.

Nieder, A. and Merten, K. (2007). A labeled-line code for small and large numerosities in the monkey prefrontal cortex. J Neurosci, in press

Page, R., Izquierdo, E., Saal, A., Codnia, J. and El Hasi, C. (2004) A response time model for judging order relationship between two symbolic stimuli. Perception and Psychophysics, 66, 196-207.

Parker, A. J. and Newsome, W. T. (1998) Sense and the single neuron: probing the physiology of perception. Annual Review of Neuroscience, 21, 227-277.

Pashler, H. (1984) Processing stages in overlapping tasks: evidence for a central bottleneck. Journal of Experimental Psychology: Human Perception and Performance, 10, 358-377.

Piazza, M., Giacomini, E., Le Bihan, D. and Dehaene, S. (2003) Single-trial classification of parallel pre-attentive and serial attentive processes using functional magnetic resonance imaging. Proceedings of the Royal Society of London, B, 270(1521), 1237-1245.

Piazza, M., Izard, V., Pinel, P., Le Bihan, D. and Dehaene, S. (2004) Tuning curves for approximate numerosity in the human intraparietal sulcus. Neuron, 44, 547-555.

Piazza, M., Pinel, P. and Dehaene, S. (2006) A magnitude code common to numerosities and number symbols in human intraparietal cortex. Neuron, in press.

Pica, P., Lemer, C., Izard, V. and Dehaene, S. (2004) Exact and approximate arithmetic in an Amazonian indigene group. Science, 306(5695), 499-503.

Pinel, P., Dehaene, S., Riviere, D. and LeBihan, D. (2001) Modulation of parietal activation by semantic distance in a number comparison task. Neuroimage, 14, 1013-1026.

Pinel, P., Piazza, M., Le Bihan, D. and Dehaene, S. (2004) Distributed and overlapping cerebral representations of number, size and luminance during comparative judgments. Neuron, 41, 983-993.

Pollmann, T. and Jansen, C. (1996) The language user as an arithmetician. Cognition, 59, 219-237.

Ratcliff, R. (1988) Continuous versus discrete information processing: modeling the accumulation of partial information. Psychological Review, 95, 238-255.

Ratcliff, R. and Rouder, J. (1998) Modelling response times for two-choice decisions. Psychological Science, 9, 347-356.

Ratcliff, R. and Tuerlinckx, F. (2002) Estimating parameters of the diffusion model: approaches to dealing with contaminant reaction times and parameter variability. Psychonomic Bulletin and Review, 9, 438-481.

Reynvoet, B., Brysbaert, M. and Fias, W. (2002) Semantic priming in number naming. Quarterly Journal of Experimental Psychology A, 55, 1127-1139.

Rivera, S. M., Reiss, A. L., Eckert, M. A. and Menon, V. (2005) Developmental changes in mental arithmetic: evidence for increased functional specialization in the left inferior parietal cortex. Cerebral Cortex, 15, 1779-1790.

Roitman, J. D., Brannon, E. M. and Platt, M. (2007). Graded Coding of Numerosity in Macaque Lateral Intraparietal Area. PLoS Biol, in press.

Romo, R. and Salinas, E. (2003) Flutter discrimination: neural codes, perception, memory and decision making. Nature Reviews Neuroscience, 4, 203-218.

Salinas, E. (2006). How behavioral constraints may determine optimal sensory representations. PLoS Biol, 4(12), e387.

Sawamura, H., Shima, K. and Tanji, J. (2002). Numerical representation for action in the parietal cortex of the monkey. Nature, 415(6874), 918-922. 
Schwarz, W. (2001) The ex-Wald distribution as a descriptive model of response times. Behavioral Research Methods and Instruments in Computing, 33, 457-469.

Shadlen, M. N. and Newsome, W. T. (1998) The variable discharge of cortical neurons: implications for connectivity, computation and information coding. Journal of Neuroscience, 18, 3870-3896.

Shadlen, M. N. and Newsome, W. T. (2001) Neural basis of a perceptual decision in the parietal cortex (area LIP) of the rhesus monkey. Journal of Neurophysiology, 86, 1916-1936.

Shadlen, M. N., Britten, K. H., Newsome, W. T. and Movshon, J. A. (1996) A computational analysis of the relationship between neuronal and behavioral responses to visual motion. Journal of Neuroscience, 16, 1486-1510.

Shepard, R. N. (2001) Perceptual-cognitive universals as reflections of the world. Behavioral and Brain Sciences, 24, 581-601; discussion 652-571.

Shepard, R. N., Kilpatrick, D. W. and Cunningham, J. P. (1975) The internal representation of numbers. Cognitive Psychology, 7, 82-138.

Siegler, R. S. and Opfer, J. E. (2003) The development of numerical estimation: evidence for multiple representations of numerical quantity. Psychological Science, 14, 237-243.

Sigman, M. and Dehaene, S. (2005) Parsing a cognitive task: a characterization of the mind's bottleneck. PLoS Biology, 3, e37.

Sigman, M. and Dehaene, S. (2006) Dynamics of the central bottleneck: dual-task and task uncertainty. PLoS Biology, 4, e220.

Simon, O., Mangin, J. F., Cohen, L., Le Bihan, D. and Dehaene, S. (2002) Topographical layout of hand, eye, calculation and language-related areas in the human parietal lobe. Neuron, 33, 475-487.

Simon, O., Kherif, F., Flandin, G., Poline, J. B., Riviere, D., Mangin, J. F., Le Bihan, D. and Dehaene, S. (2004) Automatized clustering and functional geometry of human parietofrontal networks for language, space and number. Neuroimage, 23, 1192-1202.

Smith, P. L. and Ratcliff, R. (2004) Psychology and neurobiology of simple decisions. Trends in Neurosciences, 27, 161-168.

Stone, M. (1960) Models for choice reaction time. Psychometrika, 25, 251-260.

Temple, E. and Posner, M. I. (1998) Brain mechanisms of quantity are similar in 5-year-olds and adults. Proceedings of the National Academy of Sciences of the USA, 95, 7836-7841.

Tomonaga, M. and Matsuzawa, T. (2002) Enumeration of briefly presented items by the chimpanzee (Pan troglodytes) and humans (Homo sapiens). Animal Learning and Behaviour, 30, 143-157.

Usher, M. and McClelland, J. L. (2001) The time course of perceptual choice: the leaky, competing accumulator model. Psychological Review, 108, 550-592.

Van Oeffelen, M. P. and Vos, P. G. (1982) A probabilistic model for the discrimination of visual number. Perception and Psychophysics, 32, 163-170.

Verguts, T. (2006) How to compare two quantities? A computational model of flutter discrimination. Journal of Cognitive Neuroscience, in press.

Verguts, T. and Fias, W. (2004) Representation of number in animals and humans: a neural model. Journal of Cognitive Neuroscience, 16, 1493-1504.

Verguts, T., Fias, W. and Stevens, M. (2005) A model of exact small-number representation. Psychonomic Bulletin and Review, 12, 66-80.

Wagenmakers, E.-J., van der Maas, H. L. J. and Grasman, R. P. P. P. (2006) An EZ-diffusion model for response time and accuracy. Psychonomic Bulletin and Review, in press.

Wald, A. (1947) Sequential Analysis. Wiley, New York.

Walsh, V. (2003) A theory of magnitude: common cortical metrics of time, space and quantity. Trends in Cognitive Sciences, 7, 483-488.

Washburn, D. A. and Rumbaugh, D. M. (1991) Ordinal judgments of numerical symbols by macaques (Macaca mulatta). Psychological Science, 2, 190-193. 
574 NUMERICAL COGNITION

Whalen, J., Gallistel, C. R. and Gelman, R. (1999) Non-verbal counting in humans: the psychophysics of number representation. Psychological Science, 10, 130-137.

Wong, K. F. and Wang, X. J. (2006) A recurrent network mechanism of time integration in perceptual decisions. Journal of Neuroscience, 26, 1314-1328.

Wynn, K. (1992a) Addition and subtraction by human infants. Nature, 358, 749-750.

Wynn, K. (1992b) Children's acquisition of the number words and the counting system. Cognitive Psychology, 24, 220-251. 